\title{
Moment Equations with Maximum Entropy Closure for Carrier Transport in Semiconductor Devices: Validation in Bulk Silicon
}

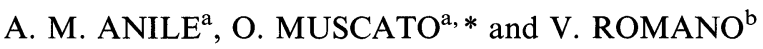 \\ a Dipartimento di Matematica, Universita' di Catania, Viale Andrea Doria 6 - 95125 Catania, Italy; \\ ${ }^{\mathrm{b}}$ Dipartimento Interuniversitario di Matematica, Politecnico di Bari, Via E. Orabona 4- 70125 Bari, Italy
}

(Received 16 December 1998; In final form 14 December 1999)

\begin{abstract}
Balance equations based on the moment method for the transport of electrons in silicon semiconductors are presented. The energy band is assumed to be described by the Kane dispersion relation. The closure relations have been obtained by employing the maximum entropy principle.

The validity of the constitutive equations for fluxes and production terms of the balance equations has been checked with a comparison to detailed Monte Carlo simulations in the case of bulk silicon.
\end{abstract}

Keywords: TCAD, VLSI, BTE, transport theory, fluid mechanics, electronic devices

\section{INTRODUCTION}

Modeling modern submicron electron devices requires an accurate description of energy transport in order to cope with high-field phenomena such as hot electron propagation, impact ionization and heat generation in the bulk material.

Also, for many applications in optoelectronics one needs to describe the transient interaction of electromagnetic radiation with carriers in complex semiconductor materials and since the characteristic times are of order of the electron momentum or energy flux relaxation times, some higher moments of the distribution function are necessarily involved. These phenomena cannot be described satisfactorily within the framework of the driftdiffusion equations (which do not comprise energy as a dynamical variable and also are valid only in the quasi-stationary limit). Therefore generalizations of the drift-diffusion equations have been sought which would incorporate energy as a dynamical variable and also would not be restricted to quasi-stationary situations. These models are loosely speaking called hydrodynamic models.

Hydrodynamic models are obtained from the infinite hierarchy of the moment equations of the

*Corresponding author. e-mail: muscato@dipmat.unict.it 
Boltzmann transport equation by a suitable truncation procedure. This requires making suitable assumptions on: (i) closing the hierarchy by finding appropriate expressions for the $N+1$ order moment in terms of the previous ones; (ii) modeling the production terms on the right hand side of the moment equations which arise from the moments of the collision terms in the Boltzmann transport equation.

One of the earliest hydrodynamic models currently used in applications was originally put forward by Blotekjaer [1] and subsequently investigated by Baccarani and Wordeman [2] and by other authors (see references in [3]). This model is implemented in simulation codes currently used in the microelectronics industry. It consists of a set of balance equations for carrier density, momentum and energy obtained from the Boltzmann transport equation in the parabolic band approximation, closed by a postulated Fourier law as constitutive equation for the heat flux. The production terms for momentum and energy are assumed to be of the relaxation type and the relaxation times are obtained by phenomenological arguments. Other models have also been investigated, some including also non parabolic effects [4-7, 9-14].

Most implemented hydrodynamic models suffer from serious theoretical drawbacks due to the ad hoc treatment of the closure problem (lacking a physically convincing motivation) and the modeling of the production terms (usually assumed to be of the relaxation type and this, as we shall see, leads to serious inconsistencies with the Onsager reciprocity relations).

In this paper we present a recently introduced moment approach in which the closure for the fluxes and also for the production terms is based on the maximum entropy principle (both for parabolic bands and also for non parabolic ones described by the Kane dispersion relation) $[13,14]$ and check the validity of the constitutive relations by Monte Carlo simulations in bulk silicon.

In Section 2 we introduce the semiclassical Boltzmann Transport Equation and the collision operator comprising the main scattering mechanisms occurring in silicon; in Section 3 we review the general framework in which the macroscopical models of charge transport in semiconductors are set.

In Sections 4 and 5 extended hydrodynamic models based on moment equations closed with the maximum entropy principle are treated in the case of parabolic band and in the case of the Kane dispersion relation and explicit closures are obtained both for high order fluxes and for the production terms. Also the consistency with the Onsager Reciprocity Principle of linear irreversible thermodynamics is shown.

In Section 6 the validity of the closure relations is checked with Monte Carlo simulations in bulk silicon and conclusions are drawn.

\section{KINETIC DESCRIPTION}

We start with the semiclassical Boltzmann Transport Equation (hereafter BTE) describing charge carrier transport in semiconductor devices $[4,15$, $16]$. In the case of electrons in a single band model (the motion of holes will be not considered in the following) it reads

$$
\frac{\partial f}{\partial t}+v^{i}(\mathbf{k}) \frac{\partial f}{\partial x^{i}}-\frac{e E^{i}}{\hbar} \frac{\partial f}{\partial k^{i}}=\mathcal{C}[f]
$$

where $f(\mathbf{x}, t, \mathbf{k})$ is the one particle distribution function giving the probability of finding an electron, in the position $\mathbf{x}$ and at time $t$, with a state belonging to a small volume of the first Brillouin zone $\mathcal{B}$ centered at the state of wave vector $\mathbf{k}$. $\mathcal{C}[f]$ describes the effects due to scattering with phonons, impurities and with other electrons. E represents the self-consistent electric field which is related to the electron distribution function through Poisson's equation

$$
\begin{gathered}
\mathbf{E}=-\nabla_{x} \phi \\
\epsilon \Delta \phi=-l\left(N_{D}-N_{A}-n\right)
\end{gathered}
$$


where $\phi$ is the electric potential, $N_{D}$ and $N_{A}$ are respectively the donor and acceptor densities, $\epsilon$ the dielectric constant and $n$ the particle density, defined as

$$
n=\int_{\mathcal{B}} f d^{3} \mathbf{k}
$$

The electron group velocity $\mathbf{v}$ is given by

$$
\mathbf{v}=\frac{1}{\hbar} \nabla_{\mathbf{k}} \mathcal{E}
$$

where $\mathcal{E}(\mathbf{k})$ is the energy of the considered conduction band structure of the crystal measured from the band minimum.

In a neighborhood of a band minimum some analytical approximation can be used for the band energy. In the parabolic approximation one assumes

$$
\mathcal{E}=\frac{\hbar^{2}|\mathbf{k}|^{2}}{2 m^{*}}
$$

with $m^{*}$ the effective electron mass (for silicon $m^{*}=0.32 m_{e}$, with $m_{e}$ the electron mass in vacuum), $\hbar \mathbf{k}$ the crystal momentum, which is assumed to vary for consistency in all $\mathcal{R}^{3}$, and $\hbar$ the Planck constant $h$ divided by $2 \pi$.

In the approximation of the Kane dispersion relation, which takes into account the non-parabolicity at high energy, $\mathcal{E}$ still depends only on $k$, the modulus of $\mathbf{k}$, but

$$
\mathcal{E}(k)[1+\alpha \mathcal{E}(k)]=\frac{\hbar^{2} k^{2}}{2 m^{*}}, \quad \mathbf{k} \in \mathcal{R}^{3},
$$

where $\alpha$ is the non parabolicity parameter (for Silicon $\alpha=0.5 \mathrm{eV}-1$ ).

Concerning the collision term, the main scattering mechanisms in a silicon semiconductor are the electron-phonon interaction, the interaction with impurities, electron-electron scatterings and interaction with stationary imperfections of the crystal as vacancies, external and internal crystal boundaries. The electron-electron scattering is important only at densities higher than those encountered in electron devices. Therefore it will not be taken into account. The scattering with impurities, imperfections and vacancies will not be considered as well.

In general the collision operator can be schematically written as

$$
\mathcal{C}[f]=\int_{\mathcal{B}}\left[P\left(\mathbf{k}^{\prime}, \mathbf{k}\right) f\left(\mathbf{k}^{\prime}\right)-P\left(\mathbf{k}, \mathbf{k}^{\prime}\right) f(\mathbf{k})\right] d^{3} \mathbf{k}^{\prime}
$$

with $P\left(\mathbf{k}, \mathbf{k}^{\prime}\right)$ the transition probability per unit time from a state $\mathbf{k}$ to a state $\mathbf{k}^{\prime}$. The first term in (6) represents the gain and the second one the loss.

In silicon electron-phonon scatterings are of elastic and inelastic types [18]. In the case of elastic scattering the transition probability writes:

$$
P\left(\mathbf{k}, \mathbf{k}^{\prime}\right)=\frac{k_{B} T_{L} \Xi_{d}^{2}}{4 \pi^{2} \hbar \rho v_{s}^{2}} \delta\left(\mathcal{E}-\mathcal{E}^{\prime}\right)
$$

where $\delta$ is the Dirac delta function, $\Xi_{d}$ is the acoustic-phonon deformation potential, $\rho$ the mass density of the material and $v_{s}$ the sound velocity of the longitudinal acoustic mode and $T_{L}$ is the lattice temperature which will be taken as constant in the following. In the case of inelastic scattering the transition probability is:

$$
\begin{aligned}
P\left(\mathbf{k}, \mathbf{k}^{\prime}\right)= & Z_{f} \frac{\left(D_{t} K\right)^{2}}{8 \pi^{2} \rho \omega}\left(n_{B}+\frac{1}{2} \mp \frac{1}{2}\right) \\
& \delta\left(\mathcal{E}^{\prime}-\mathcal{E} \mp \hbar \omega\right)
\end{aligned}
$$

where $D_{t} K$ is the deformation potential for optical phonons, $Z_{f}$ is the number of final equivalent valleys for the considered intervalley scattering (see Section 6), $\hbar \omega$ is the longitudinal optical phonon energy and $n_{B}$ is the phonon equilibrium distribution according to the Bose-Einstein statistics

$$
n_{B}=\frac{1}{\exp \left(\hbar \omega / k_{B} T_{L}\right)-1} .
$$

The double choice of sign refers to absorption and emission processes which means that we must consider the sum of the two cases. 
We observe that the nonparabolicity enters in the calculation of the moments of the collision terms only through the elementary volume in the integrals.

\section{THE MOMENT EQUATIONS}

The direct attempts to solve the system of the BTE coupled to Poisson equation, meet daunting computational difficulties and indeed, in general, solutions are available only in a stochastic sense by Monte Carlo simulations. For CAD purposes continuum models, easier to tackle from a numerical point of view, are warranted.

The macroscopic balance equations are deduced as moment equations of the Boltzmann transport equation like in gasdynamics. By multiplying Eq. (1) by a function $\psi(\mathbf{k})$ and integrating over $\mathcal{B}$, one finds the generic moment equation

$$
\begin{aligned}
\frac{\partial M_{\psi}}{\partial t} & +\int_{\mathcal{B}} \psi(\mathbf{k}) v^{i}(\mathbf{k}) \frac{\partial f}{\partial x^{i}} d^{3} \mathbf{k} \\
& -\frac{e E^{j}}{\hbar} \int_{\mathcal{B}} \psi(\mathbf{k}) \frac{\partial}{\partial k_{j}} f d^{3} \mathbf{k}=\int_{\mathcal{B}} \psi(\mathbf{k}) \mathcal{C}[f] d^{3} \mathbf{k}
\end{aligned}
$$

with

$$
M_{\psi}=\int_{\mathcal{B}} \psi(\mathbf{k}) f d^{3} \mathbf{k}
$$

the moment relative to the weight function $\psi$.

Since

$$
\begin{aligned}
\int_{\mathcal{B}} \psi(\mathbf{k}) \frac{\partial f}{\partial k^{j}} d^{3} \mathbf{k}= & \int_{\partial \mathcal{B}} \psi(\mathbf{k}) f \mathbf{n} d \sigma \\
& -\int_{\mathcal{B}} f \frac{\partial \psi(\mathbf{k})}{\partial k^{j}} d^{3} \mathbf{k}
\end{aligned}
$$

with $\mathbf{n}$ outward unit normal field on the boundary $\partial \mathcal{B}$ of the domain $\mathcal{B}$ and $d \sigma$ surface element of $\partial \mathcal{B}$, Eq. (9) becomes

$$
\begin{aligned}
\frac{\partial M_{\psi}}{\partial t} & +\frac{\partial}{\partial x^{i}} \int_{\mathcal{B}} f \psi(\mathbf{k}) v^{i}(\mathbf{k}) d^{3} \mathbf{k} \\
& +\frac{e E^{j}}{\hbar}\left[\int_{\mathcal{B}} f \frac{\partial \psi(\mathbf{k})}{\partial k_{j}} d^{3} \mathbf{k}-\int_{\partial \mathcal{B}} \psi(\mathbf{k}) f n_{j} d \sigma\right] \\
& =\int_{\mathcal{B}} \psi(\mathbf{k}) \mathcal{C}(f) d^{3} \mathbf{k} .
\end{aligned}
$$

The term

$$
\int_{\partial \mathcal{B}} \psi(\mathbf{k}) f \mathbf{n} d \sigma
$$

vanishes either when $\mathcal{B}$ is expanded to $\mathcal{R}^{3}$ (because in order to guarantee the integrability condition $f$ must tend to zero sufficiently fast as $k \mapsto \infty$ ) or when $\mathcal{B}$ is compact and $\psi(\mathbf{k})$ is periodic and continuous on $\partial B$. This latter condition is a consequence of the periodicity of $f$ on $\mathcal{B}$ and the symmetry of $\mathcal{B}$ with respect to the origin.

In the applications several choices of the weight function $\psi$ can be made and they lead to different balance equations for macroscopic quantities. If one considers as weight functions the quantities 1 , $\hbar \mathbf{k}, \mathcal{E}$ and $\mathcal{E} \mathbf{V}$, one obtains the continuity equation (indeed a term due to the generation-recombination mechanism should appear in the right hand side, but this effect is relevant for times of order $10^{-9}$ second and in most applications can be neglected because the characteristic times are of order of a fraction of picosecond), the balance equation for the crystal momentum, the balance equation for the electron energy, and the balance equation for the electron energy flux.

When the Kane dispersion relation or the parabolic approximation is considered, the explicit form of the macroscopic balance equations reads for a single valley model

$$
\frac{\partial n}{\partial t}+\frac{\partial\left(n V^{i}\right)}{\partial x^{i}}=0
$$

$$
\frac{\partial\left(n P^{i}\right)}{\partial t}+\frac{\partial\left(n U^{i j}\right)}{\partial x^{j}}+n e E^{i}=n C^{i}
$$




$$
\begin{aligned}
& \frac{\partial(n W)}{\partial t}+\frac{\partial\left(n S^{j}\right)}{\partial x^{j}}+n e V_{k} E^{k}=n C_{W}, \\
& \frac{\partial\left(n S^{i}\right)}{\partial t}+\frac{\partial\left(n F^{i j}\right)}{\partial x^{j}}+n e E_{j} G^{i j}=n C_{W}^{i},
\end{aligned}
$$

where

$$
\begin{aligned}
n= & \int_{\mathcal{R}^{3}} f d^{3} \mathbf{k} \text { is the electron density, } \\
V^{i}= & (1 / n) \int_{\mathcal{R}^{3}} f v^{i} d^{3} \mathbf{k} \text { is the average electron } \\
& \text { velocity, } \\
W= & (1 / n) \int_{\mathcal{R}^{3}} \mathcal{E}(k) f d^{3} \mathbf{k} \text { is the average electron } \\
& \text { energy, } \\
S^{i}= & (1 / n) \int_{\mathcal{R}^{3}} f v^{i} \mathcal{E}(k) d^{3} \mathbf{k} \text { is the energy flux, } \\
P^{i}= & (1 / n) \int_{\mathcal{R}^{3}} f \hbar k^{i} d^{3} \mathbf{k} \text { is the average crystal } \\
& \text { momentum, } \\
U^{i j}= & (1 / n) \int_{\mathcal{R}^{3}} f v^{i} \hbar k^{j} d^{3} \mathbf{k} \text { is the flux of crystal } \\
& \text { momentum, } \\
G^{i j}= & (1 / n) \int_{\mathcal{R}^{3}}(1 / \hbar) f\left(\partial / \partial k_{j}\right)\left(\mathcal{E} v_{i}\right) d^{3} \mathbf{k}, \\
F^{i j}= & (1 / n) \int_{\mathcal{R}^{3}} f v^{i} v^{j} \mathcal{E}(k) d^{3} \mathbf{k} \text { is the flux of energy } \\
& \text { flux, } \\
C^{i}= & (1 / n) \int_{\mathcal{R}^{3}} \mathcal{C}[f] \hbar k^{i} d^{3} \mathbf{k} \text { is the production of the } \\
& \text { crystal momentum balance equation, } \\
C_{W}= & (1 / n) \int_{\mathcal{R}^{3}} \mathcal{C}[f] \mathcal{E}(k) d^{3} \mathbf{k} \text { is the production of } \\
& \text { the energy balance equation, } \\
C_{W}^{i}= & (1 / n) \int_{\mathcal{R}^{3}} \mathcal{C}[f] v^{i} \mathcal{E}(k) d^{3} \mathbf{k} \text { is the production of } \\
& \text { the energy flux balance equation. }
\end{aligned}
$$

Analogous equations can be written for holes if a two component charge carrier model is employed.

\section{THE CLOSURE WITH THE MAXIMUM ENTROPY PRINCIPLE: THE FLUXES}

\subsection{The Maximum Entropy Principle}

The moment equations do not constitute a set of closed relations because of the fluxes and production terms. Therefore constitutive assumptions must be prescribed.

If we assume as fundamental variables $n, V^{i}, W$ and $S^{i}$, which have a direct physical interpretation, the closure problem consists in expressing $P^{i}, U^{i j}$, $F^{i j}$ and $G^{i j}$ and the moments of the collision term $C^{i}, C_{W}$ and $C_{W}^{i}$ as functions of $n, V^{i}, W$ and $S^{i}$.
First of all we stress that the role of the mean velocity $V^{i}$ here is radically different from that played in gas dynamics. In fact, for a simple gas the explicit dependence of fluxes on the velocity can be predicted by requiring galilean invariance of the constitutive functions. Instead Eqs. (11)(14) are not valid in an arbitrary galilean reference frame, but they hold only in a frame where the crystal is at rest (in the applications it can be considered as inertial and it is possible to neglect the inertial forces). Therefore $V^{i}$ is the velocity relative to the crystal and the dependence on it in the constitutive functions cannot be removed by a galilean transformation.

The Maximum Entropy Principle (hereafter MEP) leads to a systematic way for obtaining constitutive relations on the basis of information theory (see [19-23] for a review).

According to the MEP if a given number of moments $M_{A}$ are known, the distribution function $f_{M E}$ which can be used to evaluate the unknown moments of $f$, corresponds to the extremal of the entropy functional under the constraints that it yields exactly the known moments $M_{A}$

$$
\int_{\mathcal{R}^{3}} \psi_{A} f_{M E} d^{3} \mathbf{k}=M_{A}
$$

Since the electrons interact with the phonons describing the thermal vibrations of the ions placed at the points of the crystal lattice, in principle we should deal with a two component system (electrons and phonons). However, if one consider the phonon gas as a thermal bath at constant temperature $T_{L}$, only the electron component of the entropy must be maximized. Moreover, by considering the electron gas as sufficiently dilute, one can take for the electron gas the expression of the entropy obtained as limiting case of that arising in the Fermi statistics

$$
s=-k_{B} \int_{\mathcal{R}^{3}}(f \log f-f) d^{3} \mathbf{k} .
$$

If we introduce the lagrangian multipliers $\Lambda_{A}$, the problem to maximize $s$ under the constraints 
(15) is equivalent to maximize

$$
s^{\prime}=\Lambda^{A} M_{A}-s,
$$

the Legendre transform of $s$, without constraints,

$$
\delta s^{\prime}=0 .
$$

This gives

$$
\left[\log f+\frac{\Lambda_{A} \psi^{A}}{k_{B}}\right] \delta f=0
$$

Since the latter relation must hold for arbitrary $\delta f$, it follows

$$
f_{M E}=\exp \left[-\frac{1}{k_{B}} \Lambda_{A} \psi^{A}\right] .
$$

If $n, V^{i}, W$ and $S^{i}$ are assumed as fundamental variables, then

$$
\psi^{A}=(1, \mathbf{v}, \mathcal{E}, \mathcal{E} \mathbf{v})
$$

and

$$
\Lambda_{A}=\left(\lambda, k_{B} \lambda_{i}, k_{B} \lambda^{W}, k_{B} \lambda_{i}^{W}\right)
$$

with $\lambda$ lagrangian multiplier relative to the density $n, \lambda^{W}$ lagrangian multiplier relative to the energy $W, \lambda_{i}$ lagrangian multiplier relative to the velocity $V^{j}$ and $\lambda_{i}^{W}$ lagrangian multiplier relative to the energy flux $S^{j}$. Therefore the maximum entropy distribution function reads

$$
\begin{aligned}
f_{M E}=\exp [- & \frac{1}{k_{B}} \lambda+\lambda^{W} \mathcal{E} \\
& \left.\left.+\lambda_{i} v^{i}+\lambda_{i}^{W} v^{i} \mathcal{E}\right)\right],
\end{aligned}
$$

with $\Lambda_{A}$ functions of the moments $M_{A}$.

In order to get the dependence of the $\Lambda_{A}$ 's from the $M_{A}$, one has to invert the constraints (15). We will show as to get a suitable solution to this inversion problem in an asymptotic sense.

At equilibrium the distribution function is isotropic

$$
f_{E Q}=\exp \left[-\left(\frac{1}{k_{B}} \lambda_{E}+\frac{\mathcal{E}}{k_{B} T_{L}}\right)\right],
$$

that is at equilibrium

$$
\lambda_{E}^{W}=\frac{1}{k_{B} T_{L}}, \quad \lambda_{E}^{i}=0 \quad \lambda_{E}^{i W}=0 .
$$

Monte Carlo simulations for electron transport in silicon show that the anisotropy of $f$ is small $[6,7$, 17] even far from equilibrium.

Upon such a consideration we make the ansatz of small anisotropy for $f_{M E}$. Formally we introduce a small anisotropy parameter $\delta$, assume that the multipliers are analytic in $\delta$ and expand them around $\delta=0$ up to second order by taking into account the representation theorems for isotropic functions,

$$
\begin{gathered}
\lambda=\lambda^{(0)}+\delta^{2} \lambda^{(2)}, \\
\lambda^{W}=\lambda^{W(0)}+\delta^{2} \lambda^{W(2)}, \\
\lambda_{i}=\delta \lambda_{i}^{(1)}, \\
\lambda_{i}^{W}=\delta \lambda_{i}^{W(1)} .
\end{gathered}
$$

Therefore $f_{M E}$ can be written as

$$
\begin{aligned}
f_{M E}= & \exp \left(-\frac{\lambda^{(0)}}{k_{B}}-\lambda^{W(0)} \mathcal{E}\right) \\
& {\left[1-\delta x+\delta^{2}\left(\frac{x^{2}}{2}-\frac{\lambda^{(2)}}{k_{B}}-\lambda^{W(2)} \mathcal{E}\right)\right], }
\end{aligned}
$$

with $x=\lambda_{i}^{(1)} v^{i}+\lambda_{i}^{W(1)} v^{i} \mathcal{E}$.

We remark that $\lambda^{(0)}$ and $\lambda^{W(0)}$ are not the equilibrium part of $\lambda$ and $\lambda^{W}$, but the part arising when $f_{M E}$ is isotropic.

In order to get the expressions of the $\Lambda$ 's in terms of the $M_{A}$ we have to invert the following equations:

$$
\begin{gathered}
n=\int_{\mathcal{R}^{3}} f_{M E} d^{3} \mathbf{k}, \\
n W=\int_{\mathcal{R}^{3}} \mathcal{E} f_{M E} d^{3} \mathbf{k}, \\
n V^{i}=\int_{\mathcal{R}^{3}} v^{i} f_{M E} d^{3} \mathbf{k}, \\
n S^{i}=\int_{\mathcal{R}^{3}} v^{i} \mathcal{E} f_{M E} d^{3} \mathbf{k} .
\end{gathered}
$$


By retaining only the terms up to second order in $\delta$, from the constraints $(25)-(28)$, we get the following algebraic system $\left(V_{i}\right.$ and $S_{\mathrm{i}}$ are consistently considered as terms of order $\delta$ )

$$
\begin{aligned}
& n=\exp \left[-\left(\frac{1}{k_{B}} \lambda^{(0)}\right)\right] \int_{\mathcal{R}^{3}} \exp \left[-\left(\lambda^{W(0)} \mathcal{E}\right)\right] d^{3} \mathbf{k} \\
& W=\exp \left[-\left(\frac{1}{k_{B}} \lambda^{(0)}\right)\right] \int_{\mathcal{R}^{3}} \mathcal{E} \exp \left[-\left(\lambda^{W(0)} \mathcal{E}\right)\right] d^{3} \mathbf{k}
\end{aligned}
$$

$$
\begin{aligned}
& 0=\int_{\mathcal{R}^{3}} \exp \left(\lambda^{W(0)} \mathcal{E}\right) \\
& {\left[\frac{1}{k_{B}} \lambda^{(2)}+\lambda^{W(2)} \mathcal{E}-\frac{x^{2}}{2}\right]} \\
& 0=\int_{\mathcal{R}^{3}} \mathcal{E} \exp \left(\lambda^{W(0)} \mathcal{E}\right) \\
& {\left[\frac{1}{k_{B}} \lambda^{(2)}+\lambda^{W(2)} \mathcal{E}-\frac{x^{2}}{2}\right],} \\
& n V^{i}=-\int_{\mathcal{R}^{3}} v^{i} \exp \left(\lambda^{W(0)} \mathcal{E}\right) x d^{3} \mathbf{k} \\
& n S^{i}=-\int_{\mathcal{R}^{3}} v^{i} \mathcal{E} \exp \left(\lambda^{W(0)} \mathcal{E}\right) x d^{3} \mathbf{k} .
\end{aligned}
$$

Since $\mathcal{E}$ is a isotropic function of $k$, in order to solve the system for the multipliers it is computationally convenient to express $d^{3} \mathbf{k}$ in terms of $\mathcal{E}$ and the elementary volume of solid angle $d \Omega$,

$$
\begin{aligned}
& d^{3} \mathbf{k}=k^{2} d k d \Omega \\
& =\frac{m^{*}}{\hbar^{3}} \sqrt{2 m^{*} \mathcal{E}(1+\alpha \mathcal{E})}(1+2 \alpha \mathcal{E}) d \mathcal{E} d \Omega .
\end{aligned}
$$

Equations (29), (30) decouple from the other equations and explicitly read

$$
\begin{array}{r}
n=4 \\
\hbar^{3} \exp \left(-\frac{\lambda^{(0)}}{k_{B}}\right) \int_{l}^{\infty} \exp \left(-\lambda^{W(0)} \mathcal{E}\right) \\
m^{*} \sqrt{2 m^{*} \mathcal{E}(1+\alpha \mathcal{E})} \times(1+2 \alpha \mathcal{E}) d \mathcal{E},
\end{array}
$$

$W=\frac{\int_{0}^{\infty} \mathcal{E} \sqrt{\mathcal{E}(1+\alpha \mathcal{E})}(1+2 \alpha \mathcal{E}) \exp \left(-\lambda^{W(0)} \mathcal{E}\right) d \mathcal{E}}{\int_{0}^{\infty} \sqrt{\mathcal{E}(1+\alpha \mathcal{E})}(1+2 \alpha \mathcal{E}) \exp \left(-\lambda^{W(0)} \mathcal{E}\right) d \mathcal{E}}$

Relation (36) shows that $\lambda^{W(0)}$ depends only on $W$. The analytical inversion of Eq. (36) is rather involved and we have resorted to a numerical inversion.

The knowledge of $\lambda^{W(0)}$ allows us to get the constitutive functions for the other lagrangian multipliers.

Relation (35) gives $\lambda^{(0)}$, which essentially plays the role of a normalization factor

$$
\frac{\lambda^{(0)}}{k_{B}}=-\log \left(\frac{\hbar^{3} n}{4 \pi m^{*} \sqrt{2 m^{*}} d_{0}}\right),
$$

with

$$
d_{0}=\int_{0}^{\infty} \sqrt{\mathcal{E}(1+\alpha \mathcal{E})}(1+2 \alpha \mathcal{E}) \exp \left(-\lambda^{W(0)} \mathcal{E}\right) d \mathcal{E}
$$

Explicit expressions for the second order corrections to $\lambda$ and $\lambda^{W}$, are found in [13] and have the form:

$$
\begin{aligned}
& \frac{\lambda^{(2)}}{k_{B}}=\alpha_{1} \mathbf{V} \cdot \mathbf{V}+2 \alpha_{2} \mathbf{S} \cdot \mathbf{V}+\alpha_{3} \mathbf{S} \cdot \mathbf{S}, \\
& \lambda^{W(2)}=\alpha_{4} \mathbf{V} \cdot \mathbf{V}+2 \alpha_{5} \mathbf{S} \cdot \mathbf{V}+\alpha_{6} \mathbf{S} \cdot \mathbf{S}
\end{aligned}
$$

The lagrangian multiplier $\lambda_{i}$ and $\lambda_{i}^{W}$ have instead the form

$$
\left(\begin{array}{c}
\lambda_{i} \\
\lambda_{i}^{W}
\end{array}\right)=\left(\begin{array}{ll}
b_{11} & b_{12} \\
b_{12} & b_{22}
\end{array}\right)\left(\begin{array}{c}
V_{i} \\
S_{i}
\end{array}\right)
$$

The values of the $\alpha$ 's and those of $b_{i j}$ are given in [13].

\subsection{The Constitutive Equations for Fluxes}

Once the lagrangian multipliers are expressed as functions of the fundamental variables, the constitutive equations for fluxes can be obtained by using the distribution function given by the 
maximum entropy principle. First we observe that by the definition of $P^{i}$, it follows

$$
P^{i}=m^{*}\left(V^{i}+2 \alpha S^{i}\right),
$$

while for the other tensorial quantities up to second order terms the constitutive equations are of the form

$$
\begin{gathered}
U_{i j}=U_{i j}^{(0)}+\delta^{2} U_{i j}^{(2)}, \\
F_{i j}=F_{i j}^{(0)}+\delta^{2} F_{i j}^{(2)}, \\
G_{i j}=G_{i j}^{(0)}+\delta^{2} G_{i j}^{(2)} .
\end{gathered}
$$

Concerning the tensor $U^{i j}$ at the zero order we have

$$
U^{i j(0)}=U^{(0)} \delta^{i j}
$$

with

$$
U^{(0)}=\frac{2}{3 d_{0}} \int_{0}^{\infty}[\mathcal{E}(1+\alpha \mathcal{E})]^{3 / 2} \exp \left(-\lambda^{W(0)} \mathcal{E}\right) d \mathcal{E}
$$

For $U^{i j(2)}$ we get

$$
\begin{aligned}
U^{i j(2)}= & \left(\delta_{1} \mathbf{V} \cdot \mathbf{V}+2 \delta_{2} \mathbf{S} \cdot \mathbf{V}+\delta_{3} \mathbf{S} \cdot \mathbf{S}\right) \delta^{i j} \\
& +\delta_{4} V^{i} V^{j}+2 \delta_{5} V^{(i} S^{j)}+\delta_{6} S^{i} S^{j} .
\end{aligned}
$$

The coefficients $\delta_{k}$ are given in [13].

Similar calculation can be performed for $F_{i j}$ and $G_{i j}[14]$

\subsection{Parabolic Band Approximation}

In this section we shall consider the limiting case $\alpha \mapsto 0$. The aim is twofold. On one hand we will be able to get explicit formulas for the coefficients appearing in the constitutive equations, on the other hand it will be possible to have a comparison with previous hydrodynamic models. Moreover, since the difference of the results between the parabolic and Kane's dispersion relation should be small, at least at low energies, the results presented here can be useful to check the numerical evaluation of the previously obtained constitutive equations.

Concerning the lagrangian multipliers one has

$$
\begin{gathered}
\frac{\lambda}{k_{B}}=-\log \frac{n}{\left((4 / 3) \pi m^{*} W\right)^{3 / 2}} \\
+\frac{9 m^{*}}{4 W^{2}} \mathbf{V} \cdot \mathbf{S}-\frac{27 m^{*}}{20 W^{3}} \mathbf{S} \cdot \mathbf{S} \\
\lambda^{W}=\frac{3}{2 W}+\frac{21 m^{*}}{8 W^{2}} \mathbf{V} \cdot \mathbf{V}-\frac{9 m^{*}}{2 W^{3}} \mathbf{V} \cdot \mathbf{S}+\frac{81 m^{*}}{40 W^{4}} \mathbf{S} \cdot \mathbf{S}
\end{gathered}
$$

$$
\left(\begin{array}{c}
\lambda_{i} \\
\lambda_{i}^{W}
\end{array}\right)=B\left(\begin{array}{c}
V_{i} \\
S_{i}
\end{array}\right)
$$

where

$$
B=\left(\begin{array}{cc}
-\left(21 m^{*} / 4 W\right) & \left(9 m^{*} / 4 W^{2}\right) \\
\left(9 m^{*} / 4 W^{2}\right) & -\left(27 m^{*} / 20 W^{3}\right)
\end{array}\right)
$$

The distribution function given by the maximum entropy principle in this case reads

$$
\begin{aligned}
f_{M E}^{P}=\frac{n \exp \left(-\lambda^{W(0)} \mathcal{E}\right)}{\left((4 / 3) \pi m^{*} W\right)^{3 / 2}} & \\
\times\{1 & -\left(-\frac{21 m^{*}}{4 W} V_{i}+\frac{9 m^{*}}{4 W^{2}} S_{i}\right) v^{i} \\
& -\left(\frac{9 m^{*}}{4 W^{2}} V_{i}-\frac{27 m^{*}}{20 W^{3}} S_{i}\right) \mathcal{E} v^{i} \\
& +\frac{1}{2}\left[\left(-\frac{21 m^{*}}{4 W} V_{i}+\frac{9 m^{*}}{4 W^{2}} S_{i}\right) v^{i}\right. \\
& \left.+\left(\frac{9 m^{*}}{4 W^{2}} V_{i}-\frac{27 m^{*}}{20 W^{3}} S_{i}\right) \mathcal{E} v^{i}\right]^{2} \\
& -\left(\frac{9 m^{*}}{4 W^{2}} \mathbf{V} \cdot \mathbf{S}-\frac{27 m^{*}}{20 W^{3}} \mathbf{S} \cdot \mathbf{S}\right) \\
& -\left(\frac{21 m^{*}}{8 W^{2}} \mathbf{V} \cdot \mathbf{V}-\frac{9 m^{*}}{2 W^{3}} \mathbf{V} \cdot \mathbf{S}\right. \\
& \left.\left.+\frac{81 m^{*}}{40 W^{4}} \mathbf{S} \cdot \mathbf{S}\right) \mathcal{E}\right\}
\end{aligned}
$$


and the constitutive equations become

$$
\begin{gathered}
U_{i j}^{P}=\frac{2}{3} W \delta_{i j}+\left(-\frac{7}{6} m^{*} \mathbf{V} \cdot \mathbf{V}\right. \\
\left.+\frac{7 m^{*}}{5 W} \mathbf{S} \cdot \mathbf{V}-\frac{27 m^{*}}{50 W^{2}} \mathbf{S} \cdot \mathbf{S}\right) \delta^{i j} \\
+\frac{7}{2} m^{*} V^{i} V^{j}-\frac{21 m^{*}}{5 W} V^{(i} S^{j)}+\frac{81 m^{*}}{50 W^{2}} S^{i} S^{j}, \\
m^{*} F_{i j}^{P}=\frac{10}{9} W^{2} \delta_{i j}+\left(-\frac{7}{18} m^{*} W \mathbf{V} \cdot \mathbf{V}\right. \\
\left.\quad+\frac{m^{*}}{15} \mathbf{S} \cdot \mathbf{V}-\frac{9 m^{*}}{50 W} \mathbf{S} \cdot \mathbf{S}\right) \delta^{i j} \\
+\frac{77}{6} m^{*} W V^{i} V^{j}-\frac{91 m^{*}}{5} V^{(i} S^{j)} \\
+\frac{357 m^{*}}{50 W} S^{i} S^{j}, \\
G_{i j}=\frac{1}{m^{\star}}\left(U_{i j}+W \delta_{i j}\right) .
\end{gathered}
$$

\section{THE CLOSURE WITH THE MAXIMUM ENTROPY PRINCIPLE: THE PRODUCTION TERMS}

Now the $f_{M E}$ is employed to get the closure relations for the production terms by taking into account the scattering of electrons with the acoustic phonons, the non-polar optical phonons and with impurities.

\subsection{Elastic Phonon Scattering}

If we set

$$
K_{a c}=\frac{k_{B} T_{L} \Xi_{d}^{2}}{4 \pi^{2} \hbar \rho v_{s}^{2}},
$$

the collision term for elastic phonon scattering reads

$$
\begin{aligned}
\mathcal{C}[f] \approx & \mathcal{C}\left[f_{M E}\right] \\
= & \frac{n K_{a c}}{d_{0}} \sqrt{\mathcal{E}(1+\alpha \mathcal{E})}(1+2 \alpha \mathcal{E}) \exp \left(-\lambda^{(0)} \mathcal{E}\right) \\
& -\frac{4 \pi K_{a c} \sqrt{2}\left(m^{*}\right)^{3 / 2}}{\hbar^{3}} \\
& \times \sqrt{\mathcal{E}(1+\alpha \mathcal{E})}(1+2 \alpha \mathcal{E}) f_{M E} .
\end{aligned}
$$

Since the scattering is elastic one gets

$$
C_{W}=0 .
$$

Concerning the production terms of crystal momentum and energy flux, we can write

$$
\begin{aligned}
& C^{i}=c_{11}^{(a c)}(W) V_{i}+c_{12}^{(a c)}(W) S_{i}, \\
& C_{W}^{i}=c_{21}^{(a c)}(W) V_{i}+c_{22}^{(a c)}(W) S_{i} .
\end{aligned}
$$

The production matrix

$$
C^{(a c)}=\left(\begin{array}{ll}
c_{11}^{(a c)} & c_{12}^{(a c)} \\
c_{21}^{(a c)} & c_{22}^{(a c)}
\end{array}\right),
$$

is given by

$$
C^{(a c)}=A^{(a c)} B
$$

where the matrix $A^{(a c)}$ is given in [14].

\subsection{Inelastic Phonon Scattering}

For inelastic phonon scattering the collision term becomes

$$
\mathcal{C}[f] \approx \mathcal{C}\left[f_{M E}\right]=\mathcal{C}_{G}\left[f_{M E}\right]-\mathcal{C}_{L}\left[f_{M E}\right]
$$

If we set

$$
K_{n p}=\frac{\left(D_{t} K\right)^{2}}{8 \pi^{2} \rho \omega_{n p}}
$$

the gain part can be written as

$$
\begin{aligned}
\mathcal{C}_{G}\left[f_{M E}\right]= & \frac{4 \pi \sqrt{2}\left(m^{*}\right)^{3 / 2} K_{n p}}{\hbar^{3}} \exp \left(-\frac{\lambda^{(0)}}{k_{B}}\right) \\
& \left(n_{B}+\frac{1}{2} \pm \frac{1}{2}\right) \mathcal{N}_{ \pm} \exp \left( \pm \frac{\hbar \omega_{n p}}{k_{B} T_{L}}\right) \\
& \exp \left[-\lambda^{W(0)\left(\mathcal{E} \pm \hbar \omega_{n p}\right)}\right]
\end{aligned}
$$

where

$$
\begin{gathered}
N_{ \pm}=\sqrt{\left(\mathcal{E} \pm \hbar \omega_{n p}\right)\left[1+\alpha\left(\mathcal{E} \pm \hbar \omega_{n p}\right)\right]} \\
{\left[1+2 \alpha\left(\mathcal{E} \pm \hbar \omega_{n p}\right)\right]}
\end{gathered}
$$


and $v_{ \pm}$is the velocity evaluated for energy $\mathcal{E} \pm \hbar \omega_{n p}$. The loss term can be expressed as

$$
\begin{aligned}
\mathcal{C}_{L}\left[f_{M E}\right]= & \frac{4 \pi \sqrt{2}\left(m^{*}\right)^{3 / 2} K_{n p}}{\hbar^{3}} \\
& \left(n_{B}+\frac{1}{2} \mp \frac{1}{2}\right) \mathcal{N}_{ \pm} f_{M E} .
\end{aligned}
$$

One finds up to the first order in $\delta$

$$
\begin{aligned}
C_{W}= & \frac{3}{2} \frac{\bar{K}_{n p}}{d_{0}}\left(n_{B}+\frac{1}{2} \mp \frac{1}{2}\right) \\
& {\left[\exp \left( \pm \frac{\hbar \omega_{n p}}{k_{B} T_{L}} \mp \lambda^{W(0)} \hbar \omega_{n p}\right)-1\right] \eta^{ \pm} . }
\end{aligned}
$$

with

$$
\begin{gathered}
\eta^{ \pm}=\int_{\hbar \omega H(1 \pm 1)}^{\infty} \mathcal{E N}_{ \pm} \sqrt{\mathcal{E}(1+\alpha \mathcal{E})} \\
(1+2 \alpha \mathcal{E}) \exp \left(-\lambda^{W(0) \mathcal{E}}\right) d \mathcal{E} \\
\bar{K}_{n p}=\frac{8 \pi \sqrt{2}\left(m^{*}\right)^{3 / 2} K_{n p}}{3 \hbar^{3}}
\end{gathered}
$$

and $H$ is the Heviside function.

Here we do not report the quadratic correction (the interested reader is referred to [14]) because we shall employ only the linear expansion. In fact the quadratic correction is practically negligible and this justifies the well-known opinion that the energy relaxation time can be considered as function of the energy alone $[8,24]$.

At thermal equilibrium $\lambda^{W(0)}=1 / k_{B} T_{L}$ and the zeroth order term for energy production vanishes, while the second order correction can be neglected because for low energy the parabolic band approximation is valid.

The production terms of crystal momentum and energy flux have again the form

$$
\begin{gathered}
C^{i}=c_{11}^{(n p)}(W) V_{i}+c_{12}^{(n p)}(W) S_{i}, \\
C_{W}^{i}=c_{21}^{(n p)}(W) V_{i}+c_{22}^{(n p)}(W) S_{i} .
\end{gathered}
$$

The production matrix

$$
C^{(n p)}=\left(\begin{array}{ll}
c_{11}^{(n p)} & c_{12}^{(n p)} \\
c_{21}^{(n p)} & c_{22}^{(n p)}
\end{array}\right),
$$

is given by

$$
C^{(n p)}=A^{(n p)} B
$$

and the components of the matrix $A^{(n p)}$ are given in [14].

\subsection{Parabolic Band Limit}

The parabolic band limit of the closures for the production terms is recovered from the results obtained in the case of the Kane dispersion relation as $\mathcal{E} \mapsto 0$.

For the acoustic phonon scattering one finds

$$
\begin{gathered}
a_{11}^{(a c)}=\frac{32}{3} \frac{\sqrt{2 \pi} K_{a c}}{\hbar^{3}}\left(m^{*}\right)^{3 / 2}\left(\frac{2}{3} W\right)^{3 / 2} \\
a_{12}^{(a c)}=32 \frac{\sqrt{2 \pi} K_{a c}}{\hbar^{3}}\left(m^{*}\right)^{3 / 2}\left(\frac{2}{3} W\right)^{5 / 2} \\
a_{21}^{(a c)}=\frac{a_{12}^{(a c)}}{m^{*}} \\
a_{22}^{(a c)}=128 \frac{\sqrt{2 \pi m^{*}} K_{a c}}{\hbar^{3}}\left(\frac{2}{3} W\right)^{7 / 2} .
\end{gathered}
$$

Concerning the inelastic phonon scattering it is also possible to get an analytical expression for the production in terms of the Bessel functions of second kind

$$
\begin{aligned}
K_{\nu}= & \frac{\sqrt{\pi}(z / 2)^{\nu}}{\Gamma(\nu+(1 / 2))} \\
& \int_{0}^{\infty} \exp (-z \cosh t) \sin h^{2 \nu} t d t, \quad z, \nu>0,
\end{aligned}
$$

with $\Gamma$ the Gamma function. 
After some algebra one obtains the following expressions, up to the first order:

$$
\begin{aligned}
& C_{W}=\left(\frac{2}{3} W\right)^{-1 / 2} \frac{2 \sqrt{2 \pi}\left(m^{*}\right)^{3 / 2}\left(\hbar \omega_{n p}\right)^{2}}{\hbar^{3}} \\
& K_{n p}\left(n_{B}+\frac{1}{2} \mp \frac{1}{2}\right) e^{ \pm \zeta} \\
& \times\left[\exp \left( \pm \frac{\hbar \omega_{n p}}{k_{B} T_{L}} \mp 2 \zeta\right)-1\right] \\
& {\left[K_{2}(\zeta) \mp K_{1}(\zeta)\right], } \\
& a_{11}^{(n p)}= \frac{4}{3}\left(\frac{2}{3} W\right)^{-1 / 2} \frac{\sqrt{2 \pi}\left(m^{*}\right)^{3 / 2}\left(\hbar \omega_{n p}\right)^{2}}{\hbar^{3}} \\
& K_{n p}\left(n_{B}+\frac{1}{2} \mp \frac{1}{2}\right) e^{ \pm \zeta} \\
& \times\left[K_{2}(\zeta) \mp K_{1}(\zeta)\right], \\
& a_{12}^{(n p)}=\frac{4}{3} \sqrt{\frac{2}{3} W} \frac{\sqrt{2 \pi}\left(m^{*}\right)^{3 / 2}\left(\hbar \omega_{n p}\right)^{2}}{\hbar^{3}} \\
& K_{n p}\left(n_{B}+\frac{1}{2} \mp \frac{1}{2}\right) e^{ \pm \zeta} \\
& \times\left\{3 K_{2}(\zeta)+2 \zeta\left[K_{1}(\zeta) \mp K_{2}(\zeta)\right]\right\} \\
& \quad \times\left[K_{2}(\zeta)\left(12 \mp 9 \zeta+4 \zeta^{2}\right)\right. \\
& a_{22}^{(n p)}=\frac{4}{3}\left(\frac{2}{3} W\right)^{3 / 2} \frac{\sqrt{2 \pi m^{*}}\left(\hbar \omega_{n p}\right)^{2}}{\hbar^{3}} \\
& K_{n p}\left(n_{B}+\frac{1}{2} \mp \frac{1}{2}\right) e^{ \pm \zeta} \\
& a_{21}^{(n p)}=\frac{a_{12}^{(n p)}}{m^{*}}, \\
&
\end{aligned}
$$

with

$$
\zeta=\frac{3 \hbar \omega_{n p}}{4 W}
$$

Unfortunately analytical expressions are not available for the scattering with impurities. In this case one has to resort to a numerical evaluation of the coefficient of the production matrix.

\subsection{Consistency with the Onsager Reciprocity Principle in the Parabolic Band Limit}

The problem of the closure of the production terms is a delicate task: in fact in every hydrodynamic model, the physics of the material enter through these terms.

The most popular closure is that of relaxationlike approximation [2], i.e.,

$$
\begin{gathered}
C_{W}=-\frac{W-W_{0}}{\tau_{W}} \\
C^{i}=-\frac{V_{i}}{\tau_{u}} \\
C_{W}^{i}=-\frac{S_{i}}{\tau_{S}}
\end{gathered}
$$

where $W_{0}=(3 / 2) k_{B} T_{L}$ and $\tau_{W}, \tau_{u}, \tau_{S}$ are the relaxation times for energy, momentum and energy flux which must extracted by MC simulations. In principle they are functions of the energy $W$, of the electron density $n$ and of the doping density. However this functional dependence is not always good because:

- it has been shown in [24] that in the inhomogeneous case (i.e., in a $n^{+}-n-n^{+}$silicon diode), the relaxation times for momentum $\tau_{u}$ and energy flux $\tau_{S}$ versus the energy $W$ show a hysteresis loop. That does not happen for energy relaxation time $\tau_{W}$.

- it leads also to a serious inconsistency with one of the fundamental principles of Linear Irreversible Thermodynamics, the Onsager Reciprocity Principle [25] (hereafter ORP) which is not fulfilled for high electric fields [10].

It is well known that the Onsager reciprocity Principle is a manifestation of microscopic reversibility for any statistical system near thermal equilibrium: therefore any properly formulated statistical physic model should satisfy this principle.

Following the prescription of the Linear Irreversible Thermodynamics the constitutive equations between thermodynamic fluxes and forces 
are linear: the Onsager principle states that the associated matrix must be symmetric.

If one writes the balance equation for momentum (11) and for energy flux (14) in the stationary limit, by assuming that the distribution function is almost isotropic (which is borne by MC simulations [10]) and that the corresponding production terms have the form (as suggested in Sec. 4):

$$
\begin{gathered}
C_{P}^{i}=a V^{i}+b S^{i} \\
C_{W}^{i}=\tilde{a} k_{B} T_{L} V^{i}+\tilde{b} S^{i}
\end{gathered}
$$

the Onsager Reciprocity Principles states that, the following relation holds [10]:

$$
\tilde{a}+\frac{5}{2} k_{B} T_{L} \tilde{b}=\frac{5}{2} k_{B} T\left(a+\frac{7}{2} k_{B} T b\right) .
$$

Now we want to check whether the production closure equations, obtained with the Maximum Entropy Principle, satisfy the ORP. We limit ourselves to the parabolic band case because the ORP is justified near thermal equilibrium, where the non parabolic effects are negligible. The coefficients $a, b, \tilde{a}, \tilde{b}$, are given by

$$
\begin{aligned}
& a=c_{11}^{(a c)}+c_{11}^{(n p)} \\
& b=c_{12}^{(a c)}+c_{12}^{(n p)} \\
& \tilde{a}=c_{21}^{(a c)}+c_{21}^{(n p)} \\
& \tilde{b}=c_{22}^{(a c)}+c_{22}^{(n p)}
\end{aligned}
$$

where the coefficients $c_{i j}$ are given by Eqs. (60)(63), (65)-(69). It is a simple exercise to show that the ORP given by Eq. (75) is fulfilled exactly.

\section{VALIDATION OF THE CONSTITUTIVE EQUATIONS VIA MONTE CARLO SIMULATIONS}

The Monte Carlo solution of the BTE consists of a simulation of the motion of one or more electrons (and holes) inside the crystal, subject to the action of external and self consistent electric field and of given scattering mechanisms. The simulation starts by considering one or more electrons with suitable assigned initial conditions for momentum and positions. During the free flight (i.e., the time between two collisions) the external forces are made to act according to the relations:

$$
\begin{gathered}
\mathbf{v}=\frac{1}{\hbar} \nabla_{\mathbf{k}} \mathcal{E} \\
\frac{d \mathbf{k}}{d t}=\frac{q}{\hbar} \mathbf{E} .
\end{gathered}
$$

Then the scattering mechanism is chosen as responsible for the end of the free flight, according to the relative probabilities of all possible scattering mechanisms. From the differential cross section of this mechanism a new $\mathbf{k}$ state after scattering is randomly chosen as initial state of the new free flight. The Poisson equation is solved in order to obtain the new electric field and the entire process is iteratively repeated. In this way a stochastic solution to the BTE is obtained $[18,26]$. By running this procedure the history of each particle is recorded and one obtains information on some desidered quantity (e.g., the distribution function, the drift velocity, the mean energy, the stress tensor, etc.) by taking the average over the carrier histories in a time interval.

When the purpose of the analysis is the investigation of a steady-state, homogeneous case (e.g., the bulk case, with a uniform doping profile), it is sufficient in general to simulate the motion of one single electron; by assuming that the ergodicity hypothesis holds, a sufficiently long path of this sample electron will give the required information on the behaviour of the entire electron gas. When, on the contrary, the transport process under investigation is not homogeneous or is not stationary, then it is necessary to simulate a large number of electrons and follow them in their dynamic histories in order to obtain the desired information on the process of interest.

In silicon electrons which contribute mainly to the charge transport, even at high electric fields, are those of the band [18] with the lower energy: 
in the Brillouin zone this corresponds to six equivalent ellipsoidal valleys along the axis of the frame of reference centered at about $0.85 \%$ from the center of the first Brilloiun zone. The effect of non parabolicity is important at high electric fields, when electrons can reach energies far from the bottom of the conduction band.

After a collision the electron can remain in the same valley (intravalley scattering) or be drawn in another equivalent valley (intervalley scattering). In silicon the allowed electron-phonon scattering can be summarized as follows:

- scattering with intravalley acoustic phonon (elastic);

- scattering with intervalley acoustic phonons (inelastic);

- scattering with non polar optical phonons (inelastic).

For inelastic collision the values of the phonon energy $(\hbar \omega)$, the deformation potential $\left(D_{t} K\right)$ and the number of equivalent valleys $Z_{f}$ are given in Table I according to [18]. In Table II the other physical parameters for bulk silicon are reported. We have tested the constitutive equations for fluxes and production terms both for Kane dispersion relation and parabolic band limit with our MC code. Silicon was at room temperature

TABLE I Phonon energies deformation potentials and number of equivalent valleys used for silicon

\begin{tabular}{lcc}
\hline$\hbar \omega(\mathrm{meV})$ & $\left(D_{t} K\right)\left(10^{8} \mathrm{eV} / \mathrm{cm}\right)$ & $Z_{f}$ \\
\hline 12 & 0.5 & 1 \\
18.5 & 0.8 & 1 \\
19.0 & 0.3 & 4 \\
47.4 & 2.0 & 4 \\
61.2 & 11 & 1 \\
59.0 & 2.0 & 4 \\
\hline
\end{tabular}

TABLE II Values of the physical parameters of silicon

\begin{tabular}{llc}
\hline$m_{e}$ & electron rest mass & $9.109510^{-28} \mathrm{~g}$ \\
$m^{\star}$ & effective mass & $0.32 m_{e}$ \\
$T_{L}$ & lattice temperature & $300^{\circ} \mathrm{K}$ \\
$\rho_{0}$ & density & $2.33 \mathrm{~g} / \mathrm{cm}^{3}$ \\
$v_{s}$ & longitudinal sound speed & $9.1810^{5} \mathrm{~cm} / \mathrm{sec}$ \\
$\Xi_{d}$ & acoustic-phonon deformation & $9 \mathrm{eV}$ \\
& potential & \\
\hline
\end{tabular}

$\left(T_{L}=300^{\circ} \mathrm{K}\right)$ and a homogeneous electric field was frozen in the material along the $x$ direction $\left(E_{x}=5,000 \div 80,000 \mathrm{~V} / \mathrm{cm}\right)$ : we gather statistics after the transient regime, i.e., our simulation results are valid for the stationary regime.

For each electric field we evaluate $W, \mathbf{V}, \mathbf{S}$, $U^{i j}, F^{i j}, G^{i j}, C_{W}, C^{i}, C_{W}^{i}$. Since the electric field is along the $x$ axis, the tensorial variables involved have non vanishing components along this axis.

In Figure 1 we plot the percent error for $U^{11}$ both for linear and non linear closure in the parabolic band case Eq. (51); in Figure 2 we plot the same quantity in the non parabolic case. We notice that the quadratic closure is more accurate than the linear one. The comparisons for the tensor $F^{i j}$ are shown in Figures 3 and 4: the behaviour of the error is similar to that found for $U^{11}$. Significant difference in the accuracy is not found for the fluxes between parabolic and non parabolic cases. In Figure 5 we plot the percent error for $G^{11}$ in the case of Kane dispersion relation: again the agreement is good and in particular for the non linear closure.

At last we have also checked with our MC code the production closure relations obtained with maximum entropy principle. For the production only the linear closure is used. In the hydrodynamic model the six $\mathrm{X}$-valleys are considered as equivalent to a single valley. All the intravalley and intervalley scatterings have been taking into account, according to above collision mechanisms used in our MC code. The error in energy, momentum and energy-flux productions are shown respectively in Figures $6-11$. The percent error for the production term $C_{W}$ of the energy balance equation is below $15 \%$ and the agreement with MC data is better in the parabolic case (see Figs. 6 and 7). For the production $C^{1}$ of the moment balance equation the error is roughly the same (see Figs. 8 and 9). The deviation of the error with respect to the average for small values of the energy is probabily due to noise in the $\mathrm{MC}$ statistics for low electric fields. The percent error for the production term $C_{W}^{1}$ of the energy flux balance equation is shown in Figures 10 and 11: 


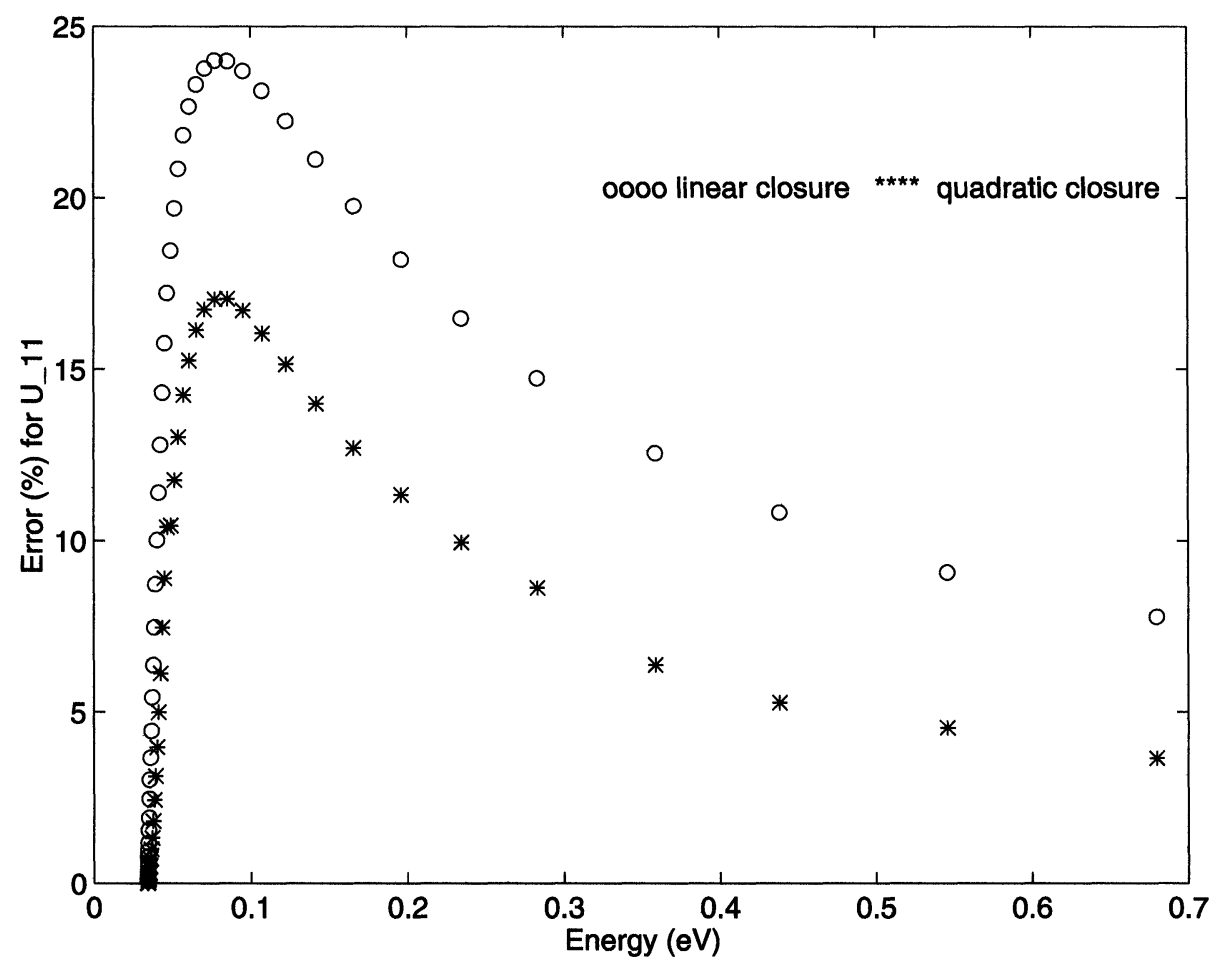

FIGURE 1 Percent error for the component $U^{11}$ of the flux of crystal momentum versus energy, in the parabolic band case.

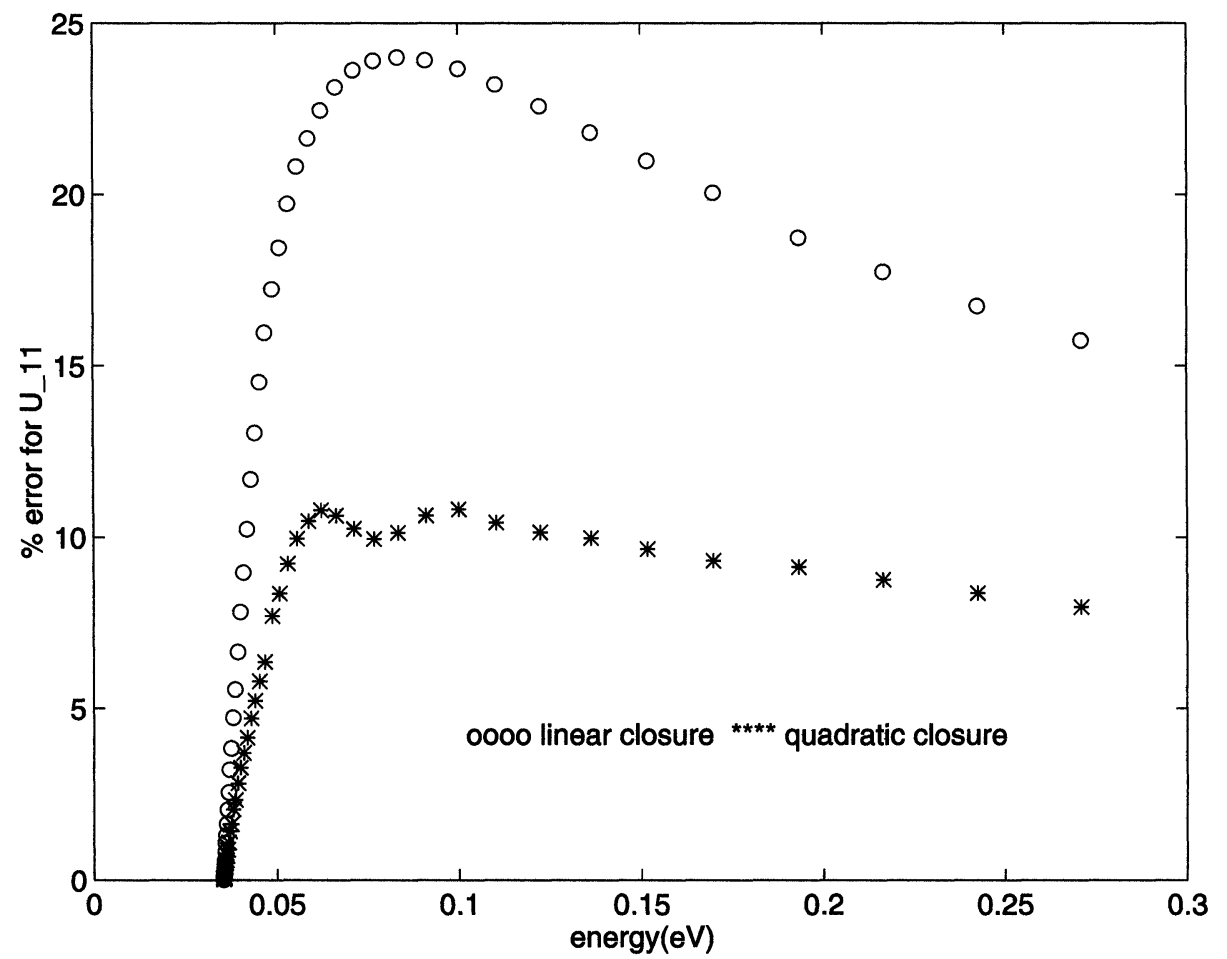

FIGURE 2 Percent error for the component $U^{11}$ of the flux of crystal momentum versus energy, in the case of the Kane dispersion relation. 


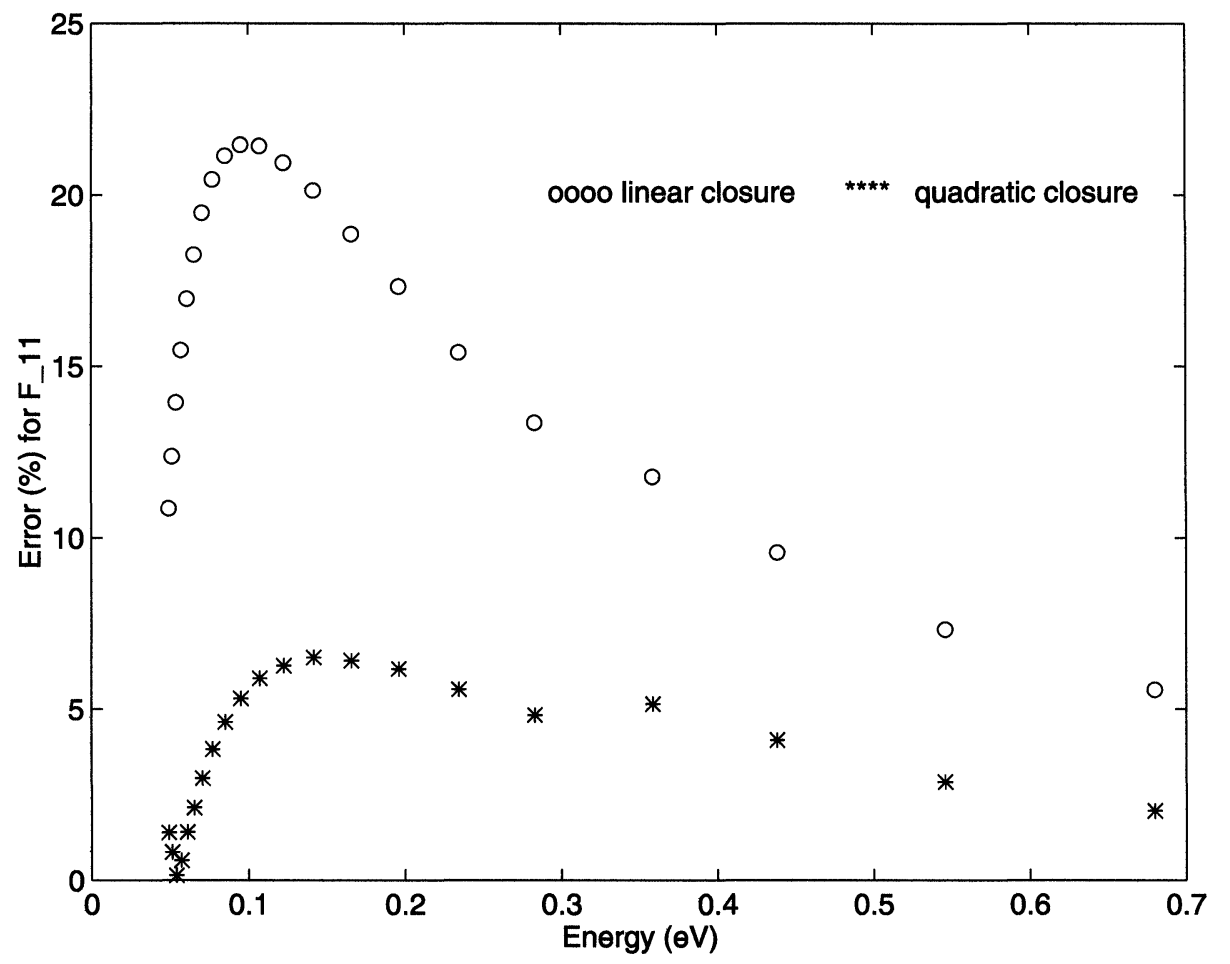

FIGURE 3 Percent error for the component $F^{11}$ of the flux of energy flux versus energy, in the parabolic band case.

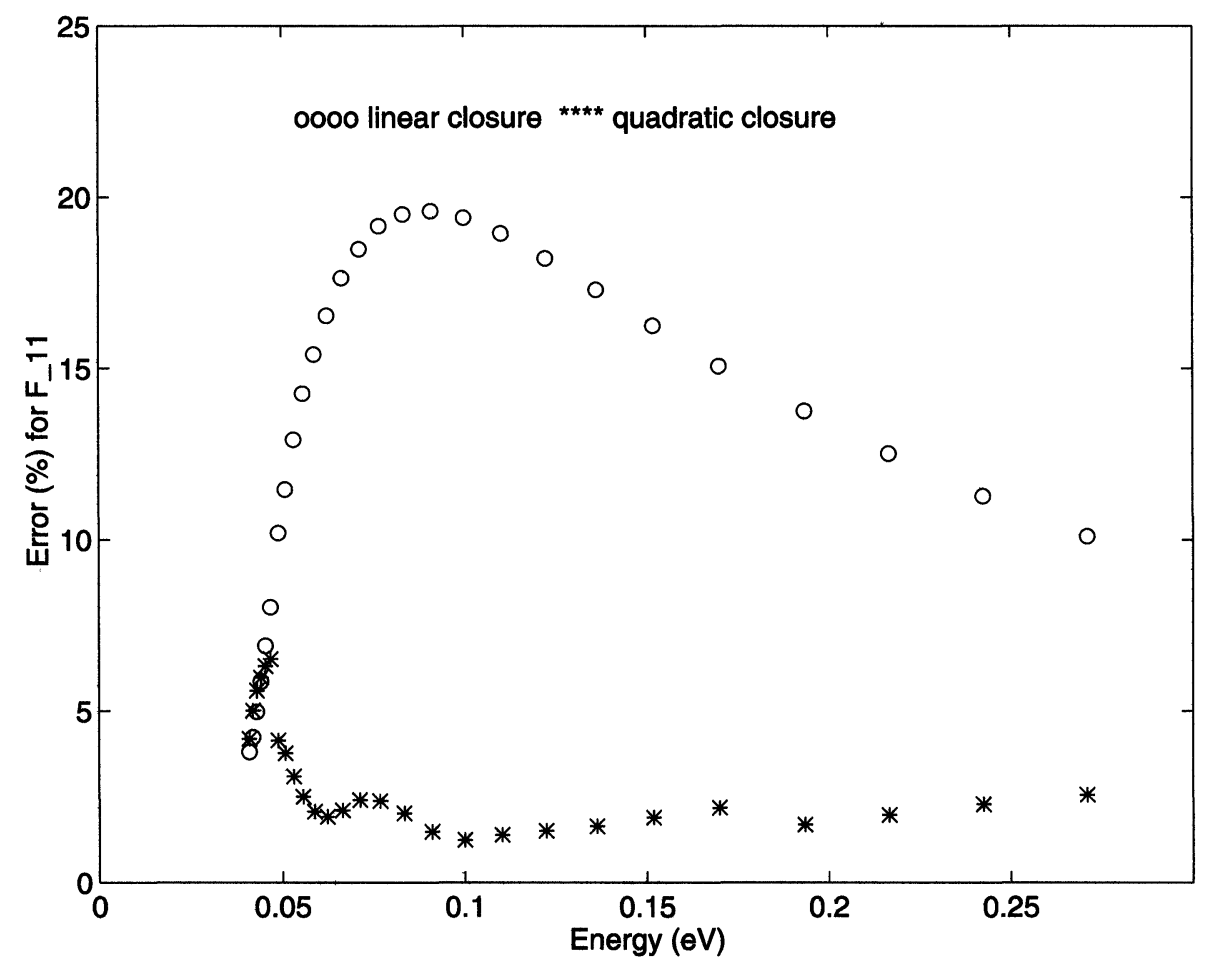

FIGURE 4 Percent error for the component $F^{11}$ of the flux of energy flux versus energy, in the case of the Kane dispersion relation. 


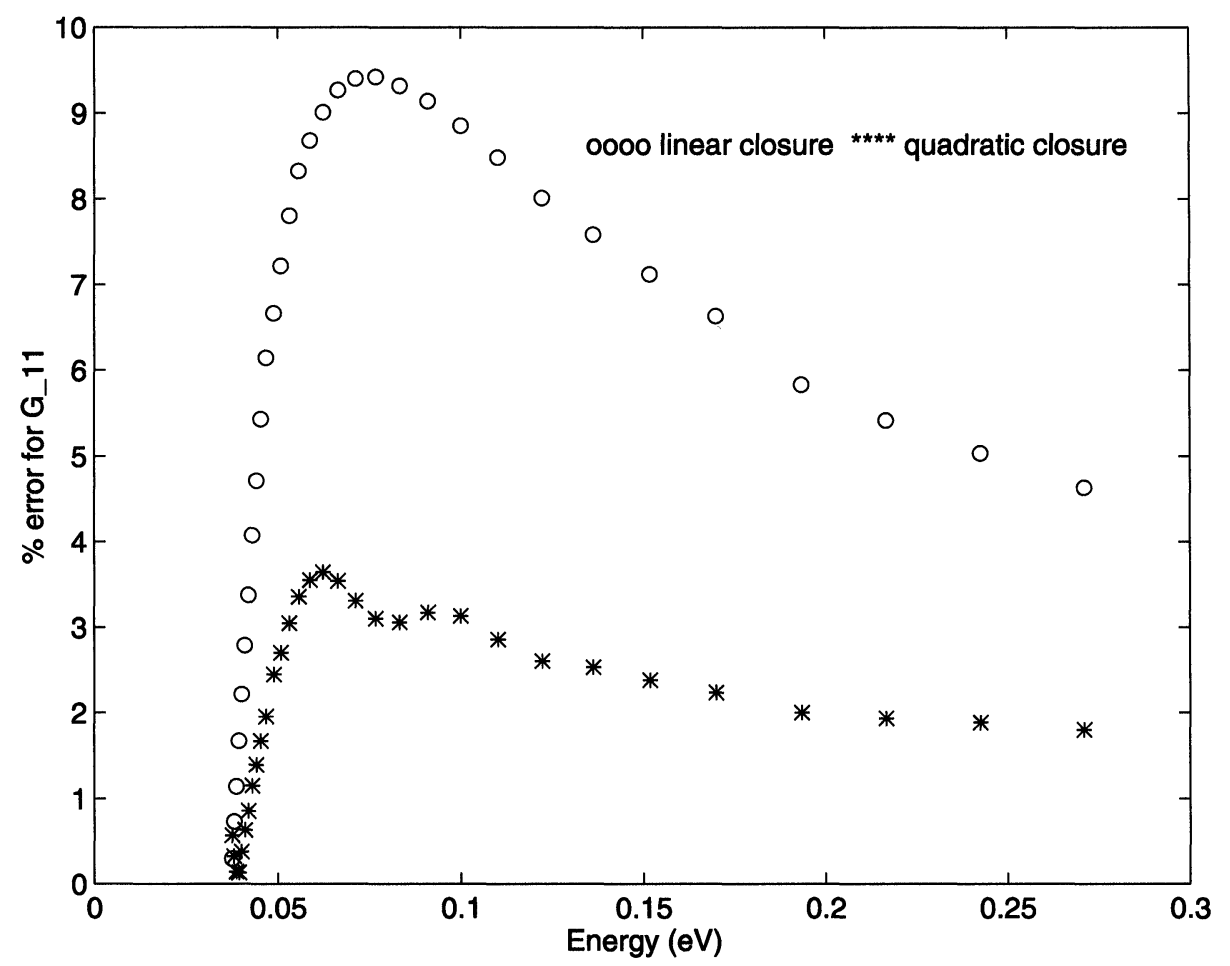

FIGURE 5 Percent error for the component $G^{11}$ versus energy, in the case of the Kane dispersion relation.

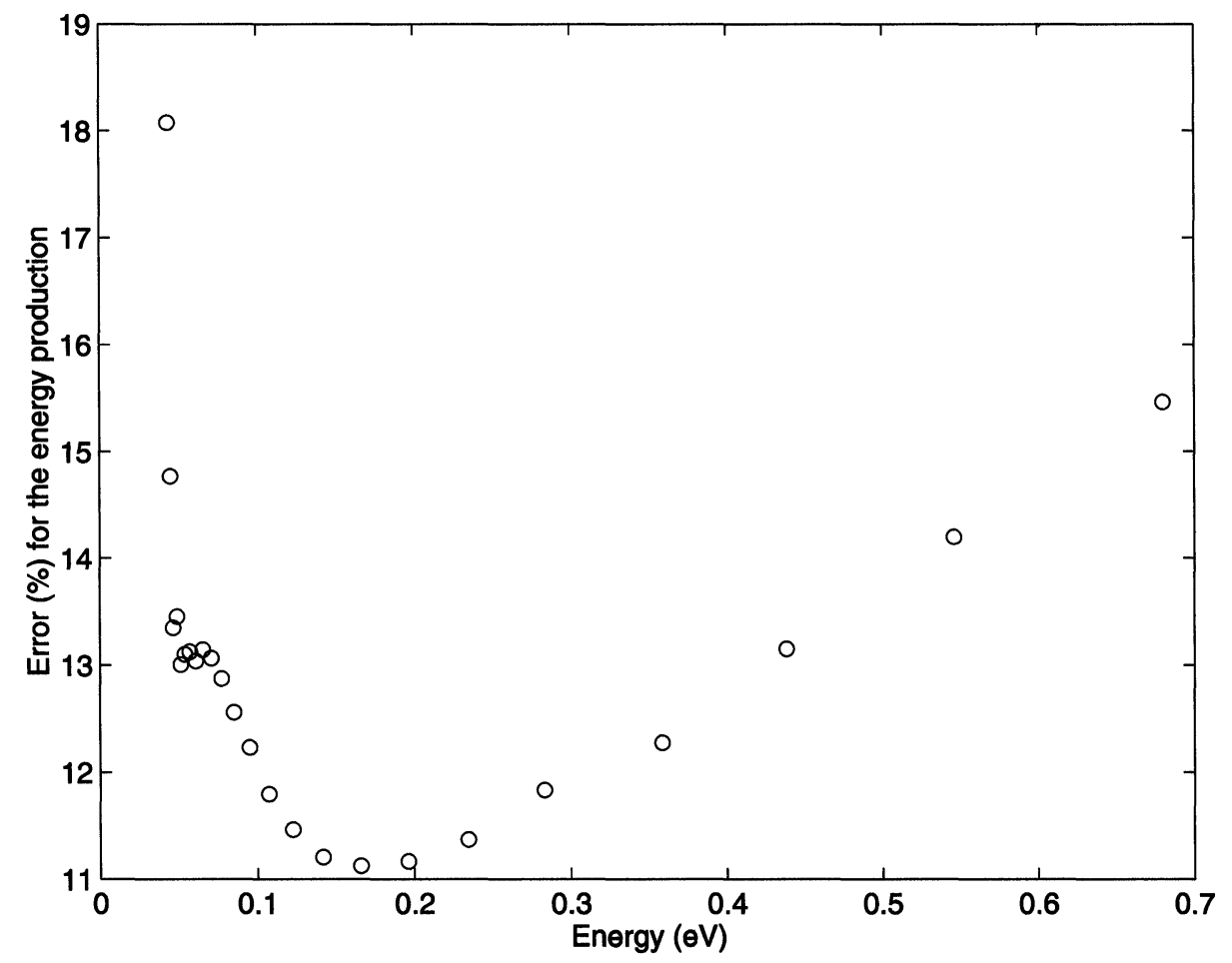

FIGURE 6 Percent error for the energy production term $C_{W}$ versus energy, in the parabolic band case. 


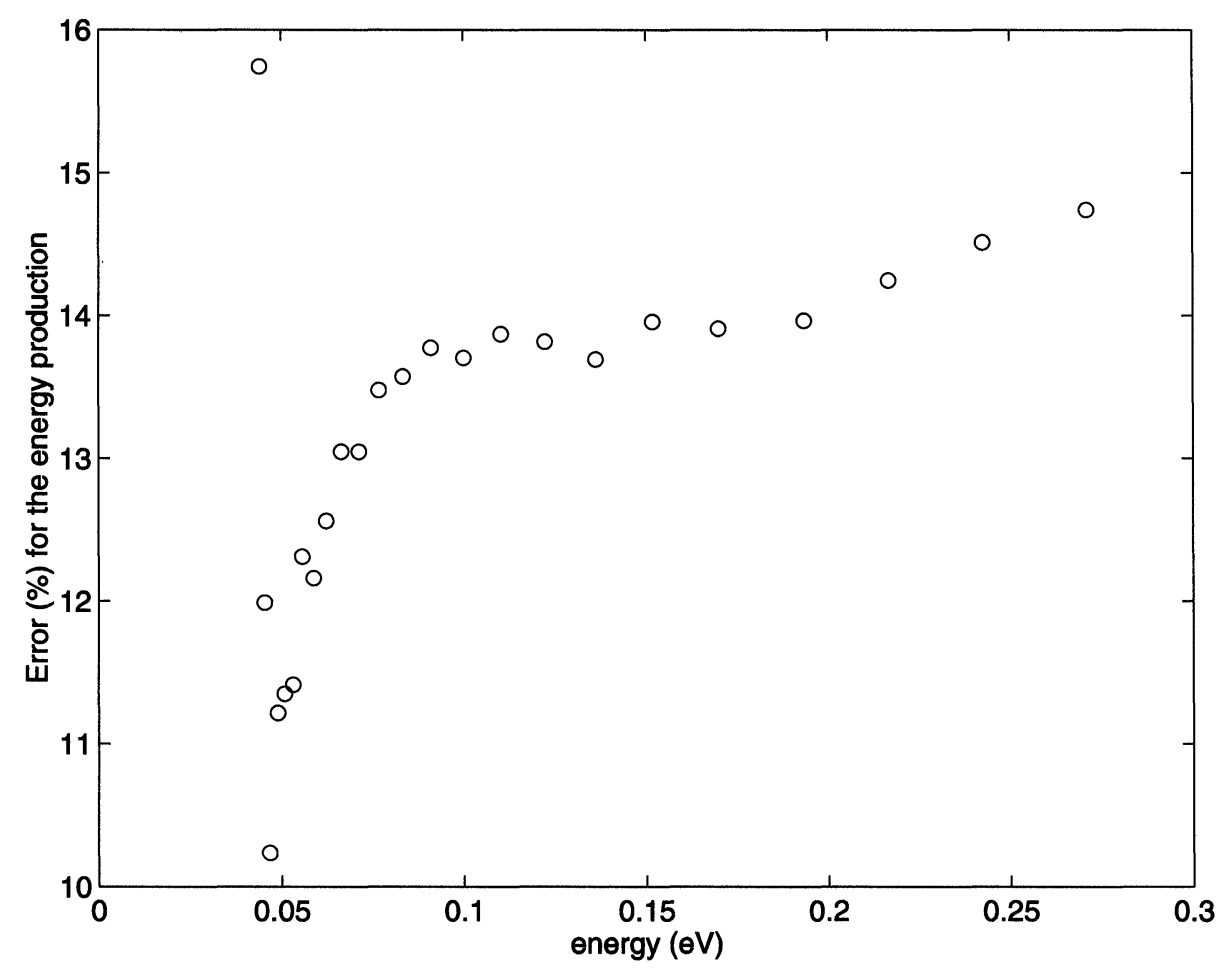

FIGURE 7 Percent error for the energy production term $C_{W}$ versus energy, in the case of the Kane dispersion relation.

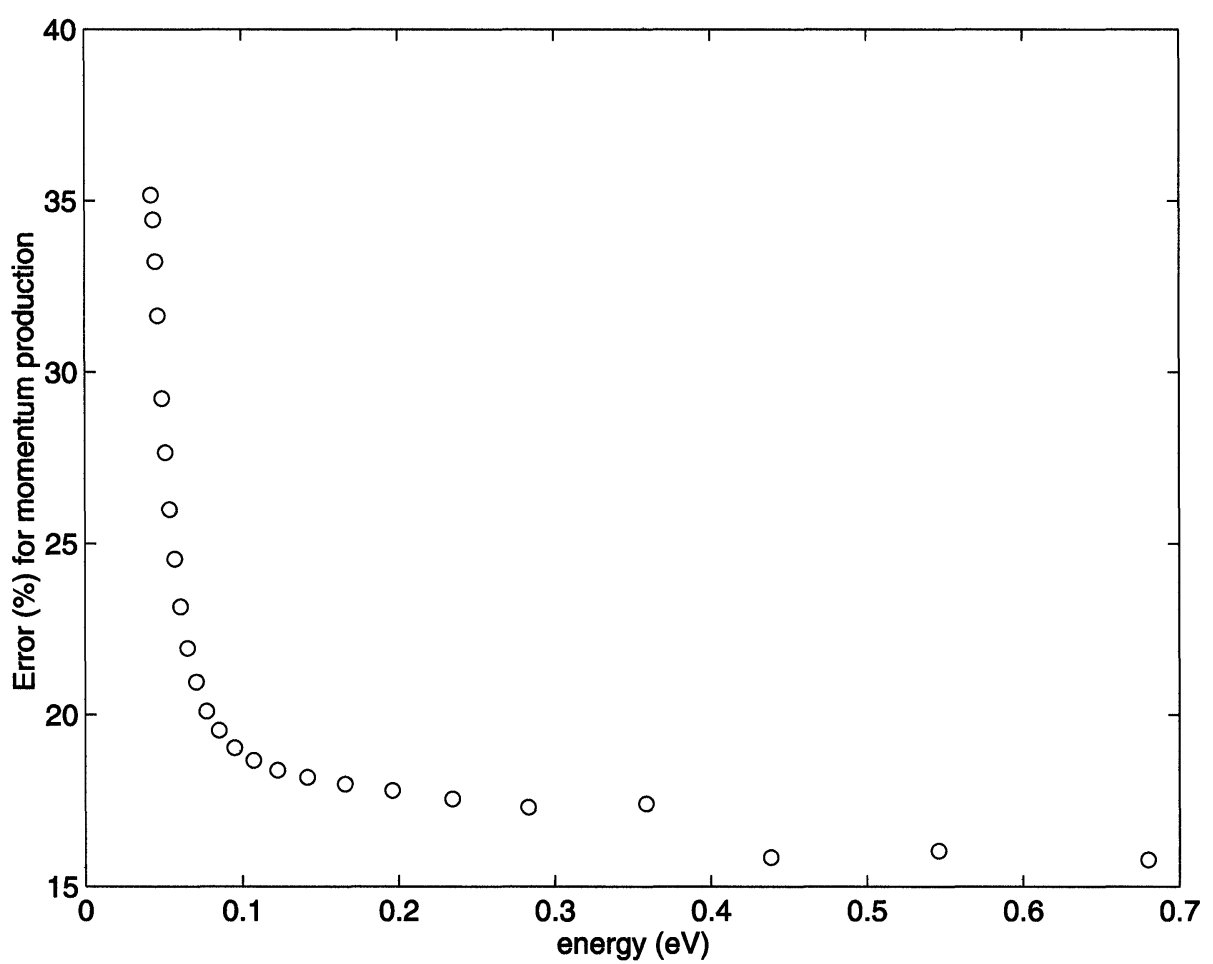

FIGURE 8 Percent error for the momentum production term $C^{1}$ versus energy, in the parabolic band case. 


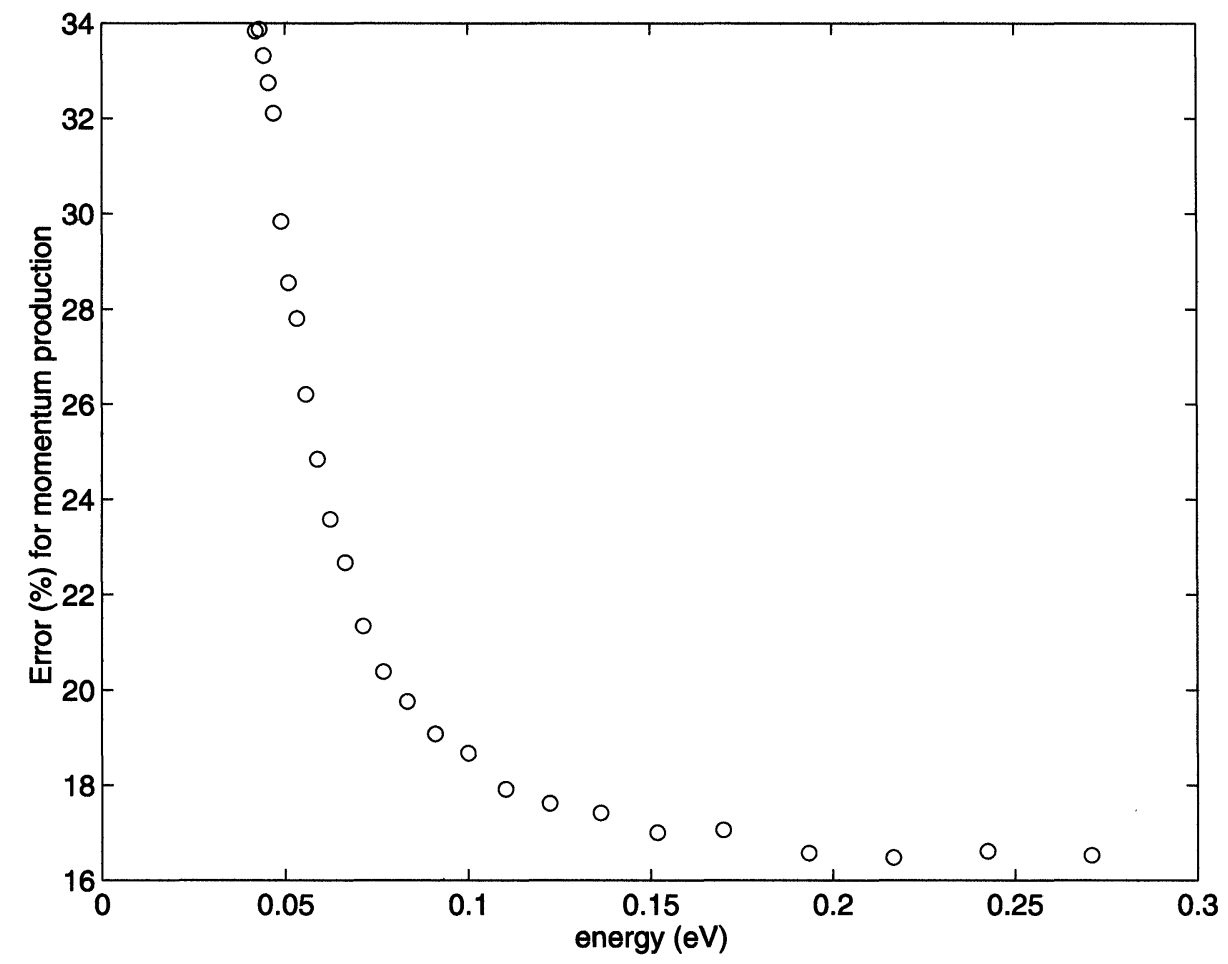

FIGURE 9 Percent error for the momentum production term $C^{1}$ versus energy, in the case of the Kane dispersion relation.

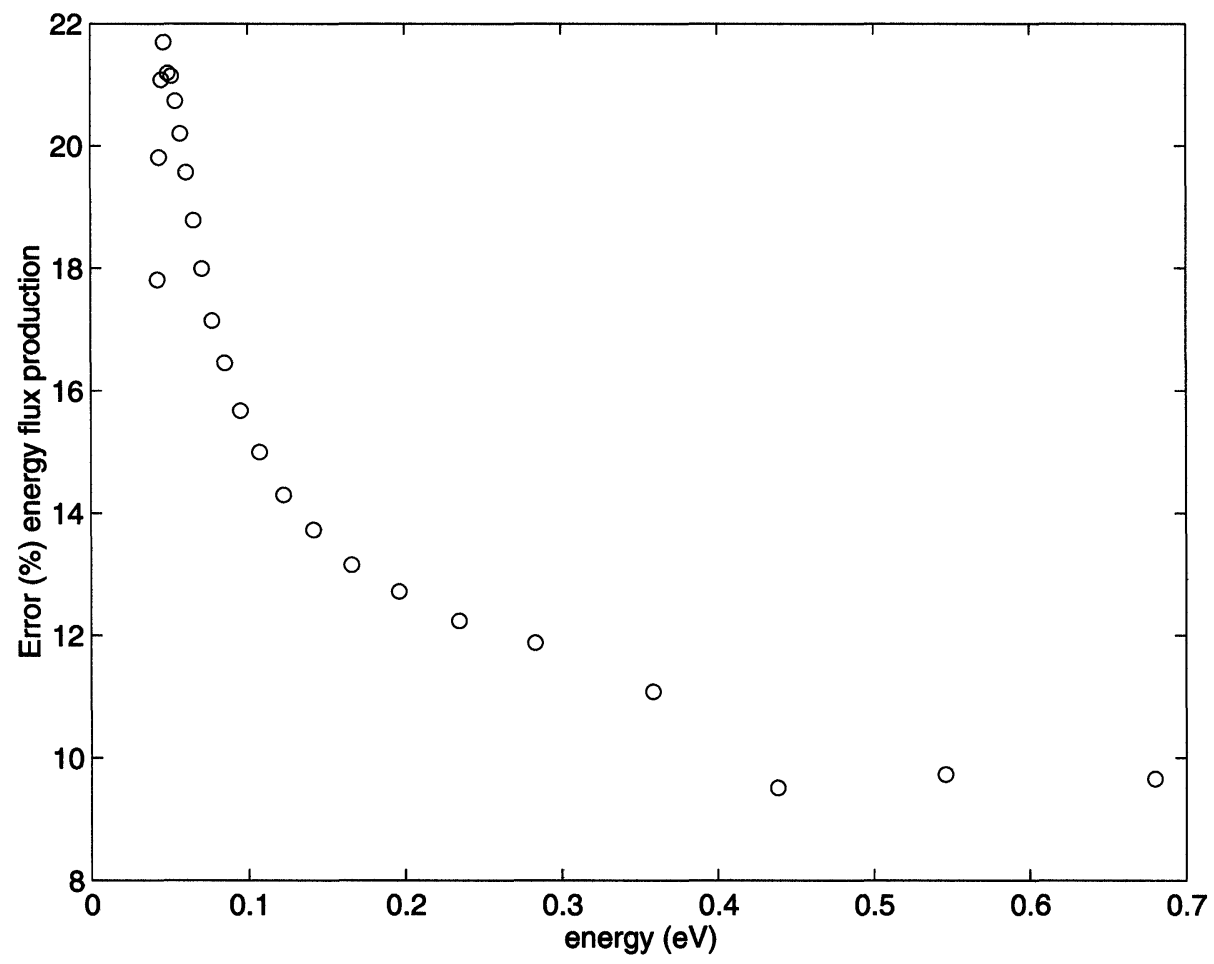

FIGURE 10 Percent error for the energy flux production term $C_{W}^{1}$ versus energy, in the parabolic band case. 


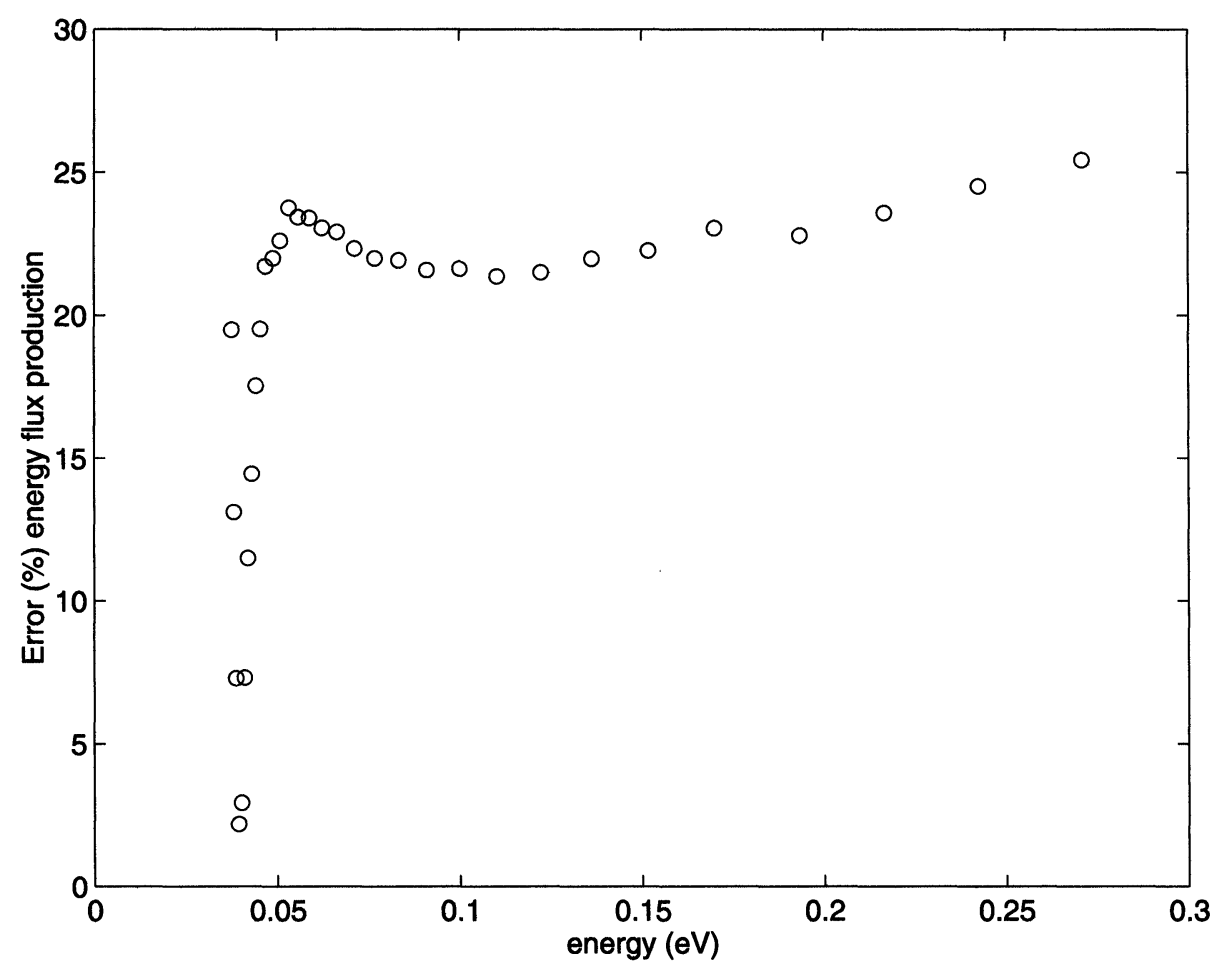

FIGURE 11 Percent error for the energy flux production term $C_{W}^{1}$ versus energy, in the case of the Kane dispersion relation.

we notice again that we have a better accuracy in the parabolic case.

\section{CONCLUSIONS}

In this paper we have checked the consistency of a hydrodynamic model for electron transport in silicon which takes into account the non parabolicity of the energy band via the Kane dispersion relation. The closure relations are based on the Maximum Entropy Principle and the constitutive equations for the production terms are in agreement with the Onsager Reciprocity Principle. A comparison with detailed MC simulations for bulk silicon has been used to test the accuracy of the model. The average error in the constitutive equations for fluxes is less then $10 \%$, whereas the average error for the production terms is less then $20 \%$. Moreover the agreement with the MC data is better in the parabolic case.
According to these results the proposed hydrodynamic model represents a viable and promising tool for simulating submicron electron devices.

\section{Acknowledgements}

This work has been supported by MURST $40 \%$ and $60 \%$, by the TMR-Network project No. ERBFRCT970157 Asympotic Methods in Kinetic Theory, by CNR project Modelli matematici per semiconduttori. Progetto speciale: modelli matematici in fluidodinamica e dinamica molecolare grant \# 96.03855.CT01.

\section{References}

[1] Blotekjaer, K. (1970). Transport equations for electron in two-valley semiconductors, IEEE Trans. on Electron Devices, ED-17, pp. 38-47.

[2] Baccarani, G. and Wordeman, M. R. (1982). An investigation on steady-state velocity overshoot in silicon, Solid-State Electronics, 29, 970-977. 
[3] Rudan, M. and Baccarani, G. (1995). On the structure and closure-condition of the hydrodynamic model, special Issue VLSI Design, 3, 115-129, Editor Jerome, J.

[4] Hänsch, W., The drift-diffusion equation and its application in MOSFET modeling, Springer-Verlag, Wien, 1991.

[5] Thoma, R., Edmunds, A., Meinerzhagen, B., Peifer, H. J. and Engl, W. (1991). Hydrodynamic Equations for Semiconductors with Nonparabolic Band Structure, IEEE Trans. Electron Devices, 38, 1343-1353.

[6] Tang, T.-W., Ramaswamy, S. and Nam, J. (1993). An Improved Hydrodynamical Transport Model for Silicon, IEEE on Electron Devices, 40, 1469-1477.

[7] Stettler, M. A., Alam, M. A. and Lundstrom, M. S. (1993). A critical examination of the assumptions underlying macroscopic transport equations for silicon device, IEEE Trans. on Electron Devices, 40(4), 733-739.

[8] Lee, S.-C. and Tang, T.-W. (1992). Transport coefficients for a silicon hydrodynamical model extracted from inhomogeneous Monte-Carlo simulation, Solid-State Electronics, 35(4), 561 - 569.

[9] Woolard, D. L., Tian, H., Trew, R. J., Littlejohn, M. A. and Kim, W. (1991). Hydrodynamic electron-transport model: Nonparabolic corrections to the streaming terms, Phys. Rev. B, 44, 11119-11132.

[10] Anile, A. M. and Muscato, O. (1995). Improved hydrodynamical model for carrier transport in semiconductors, Phys. Rev. B, 51, $16728-16740$.

[11] Anile, A. M., Romano, V. and Russo, G. (1998). Hyperbolic hydrodynamical model of carrier transport in semiconductors, VLSI Design, 8(1-4), 521-526.

[12] Anile, A. M., Romano, V. and Russo, G. (1998). Extended hydrodynamical model of carrier transport in semiconductors, to appear in SIAM J. on Appl. Math.

[13] Anile, A. M. and Romano, V. (1999). Nonparabolic band transport in semiconductors: closure of the moment equations, Cont. Mech. Thermodyn., 11, 307-325.

[14] Romano, V. (1999). Nonparabolic band transport in semiconductors: closure of the production terms to appear in Cont. Mech. Thermodynamics.

[15] Selberherr, S., Analysis and simulation of semiconductor devices, Springer-Verlag, Wien, New York, 1984.

[16] Markowich, A., Ringhofer, C. A. and Schmeiser, C. Semiconductor equations, Springer-Verlag, Wien, 1990.

[17] Anile, A. M. and Muscato, O. (1996). Extended thermodynamics tested beyond the linear regime: the case of electron transport in silicon semiconductors, Continuum Mech. Thermodyn., 8, 131-142.
[18] Jacoboni, C. and Reggiani, L. (1983). The Monte Carlo method for the solution of charge transport in semiconductors with applications to covalent materials, Rev. Mod. Phys., 55, 645-705.

[19] Müller, I. and Ruggeri, I., Rational Extended Thermodynamics, Springer-Verlag, Berlin, 1998.

[20] Jou, D., Casas-Vazquez, J. and Lebon, G., Extended irreversible thermodynamics, Springer-Verlag, Berlin, 1993.

[21] Levermore, C. D. (1995). Moment Closure Hierarchies for the Boltzmann-Poisson Equation, VLSI DESIGN, 8(1-4), 97-101.

[22] Levermore, C. D. (1996). Moment Closure Hierarchies for Kinetic Theories, J. Stat. Phys., 83, 331-407.

[23] Dreyer, W. (1987). Maximization of the entropy in nonequilibrium, J. Phys. A: Math. Gen., 20, 6505-6517.

[24] Muscato, O. (2000). Monte Carlo evaluation of the transport coefficients in a $n^{+}-n-n^{+}$silicon diode, to appear in COMPEL.

[25] de Groot, S. R. and Mazur, P. (1985). Non Equilibrium Thermodynamics (Dover Publications Inc.,), New York.

[26] Laux, S. E., Fischetti, M. V. and Frank, D. J. (1990). IBM J. Res. Develop., 34

\section{Authors' Biographies}

A. Marcello Anile is full Professor of Theoretical Mechanics at Catania University. His research interests include mathematical models for semiconductors, wave propagation.

Orazio Muscato is Assistant Professor of Theoretical Mechanics at Catania University. His research interests include mathematical models for semiconductors, Monte Carlo simulations.

Vittorio Romano is Assistant Professor of Theoretical Mechanics at Politecnico di Bari. His research interests include radiation hydrodynamics, relativistic fluids, wave propagation, numerical methods for hyperbolic systems and hydrodynamical models for semiconductors. 

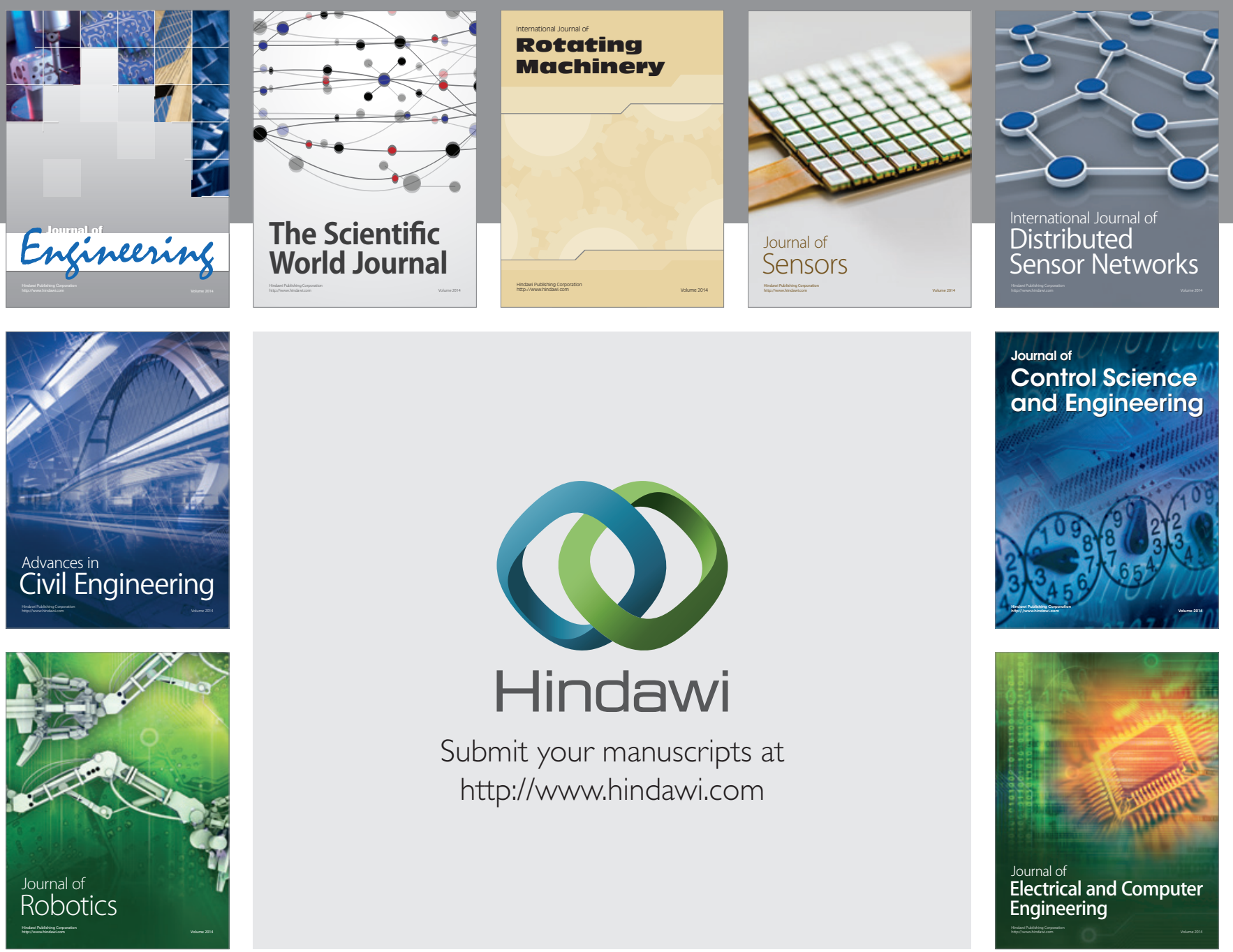

Submit your manuscripts at

http://www.hindawi.com
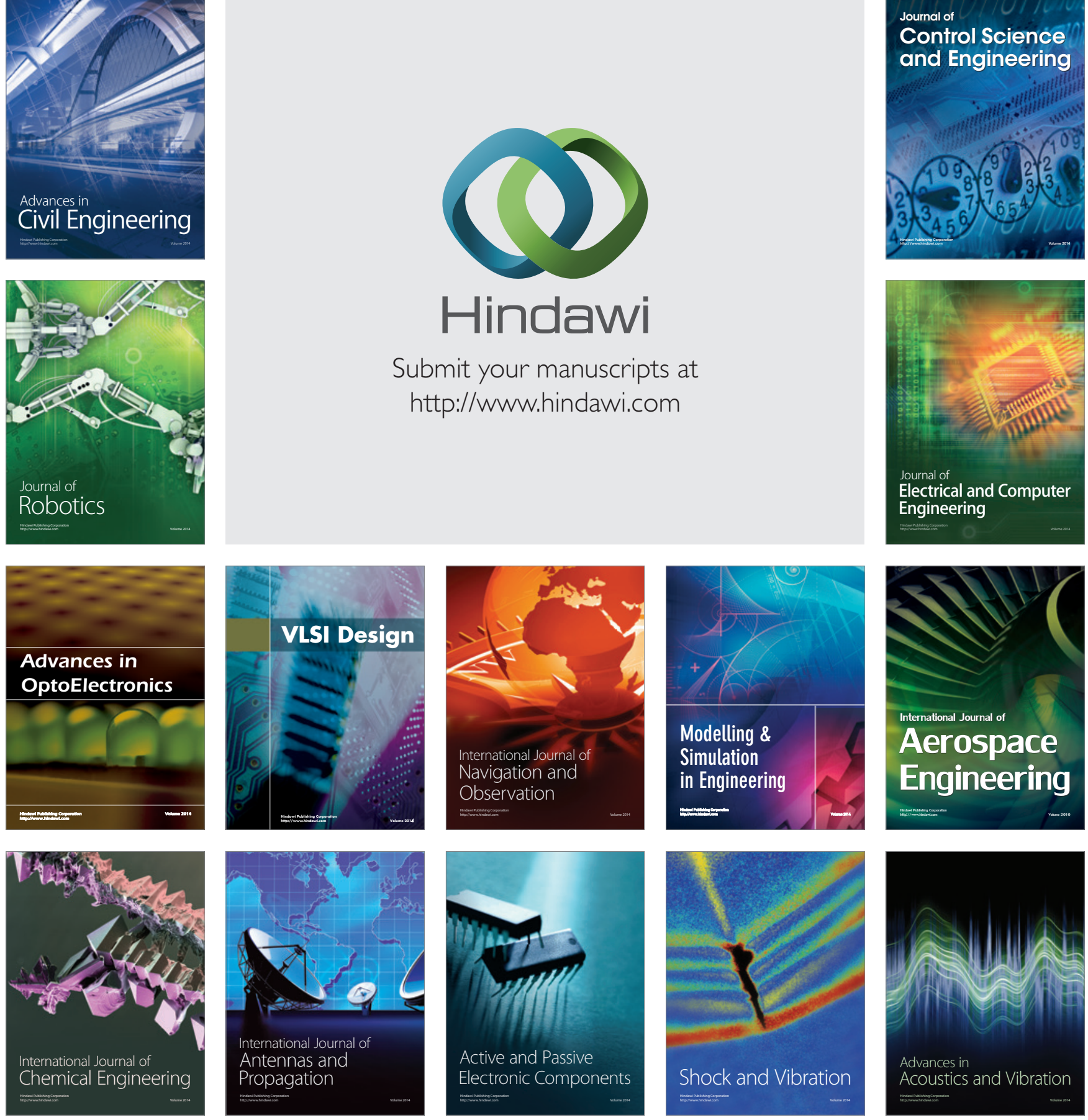